\title{
Genetic Parameters of Water and Feed Intake and Behavior Traits of Wean-to-Finish Pigs Under a Polymicrobial Natural Disease Challenge
}

\section{Jian Cheng}

lowa State University of Science and Technology: lowa State University https://orcid.org/0000-00027560-068X

Austin M. Putz

lowa State University of Science and Technology: lowa State University

John C. S. Harding

University of Saskatchewan

Michael K. Dyck

University of Alberta

Frederic Fortin

CDPQ

\section{Graham S. Plastow}

University of Alberta

Jack Dekkers ( $\square$ jdekkers@iastate.edu )

lowa State University https://orcid.org/0000-0003-1557-7577

\section{Research}

Keywords: feeding and drinking behavior, disease resilience, genetic parameters, pigs

Posted Date: March 1st, 2021

DOI: https://doi.org/10.21203/rs.3.rs-249650/v1

License: (a) (1) This work is licensed under a Creative Commons Attribution 4.0 International License. Read Full License 
1 Genetic parameters of water and feed intake and behavior traits of wean-to-

\section{finish pigs under a polymicrobial natural disease challenge}

3

4 Jian Cheng", Austin M. Putz ${ }^{*}, \dagger$, John C. S. Harding ${ }^{\ddagger}$, Michael K. Dyck ${ }^{\S}$, Frederic Fortin ${ }^{\#}$, Graham

$5 \quad$ S. Plastow ${ }^{\S}$, PigGen Canadall, and Jack C. M. Dekkers*

$6 \quad$ *Department of Animal Science, Iowa State University, Ames, IA, United States, 50011

$7 \quad{ }^{\dagger}$ Hendrix Genetics, Swine Business Unit, Boxmeer, The Netherlands, 5831 CK

8 Department of Large Animal Clinical Science, University of Saskatchewan, Saskatoon, SK,

9 Canada, S7N 5A2

$10{ }^{\S}$ Department of Agriculture, Food and Nutritional Science, University of Alberta, Edmonton, AB,

11 Canada, T6G 2R3

12 \#Centre de Développement du Porc du Québec Inc., Québec City, Canada, G1V 4M6

13 \| PigGen Canada Research Consortium, Guelph, Ontario, Canada, N1H4G8

14

15

16

17

18

19

20

21

22

23

24

25

26

27

28

29

30

31

32

33

34 


\section{ABSTRACT}

Background: The pork industry faces unprecedented challenges from disease, which increases cost of production and use of antibiotics, and reduces production efficiency, carcass quality, and

39 animal wellbeing. One solution is to improve the overall resilience of pigs to a broad array of common diseases through genetic selection. Behavioral changes in eating and drinking are usually

41 the very first clinical signs when animals are exposed to stressors such as disease. Changes in feed

42 and water intake behaviors in diseased pigs may reflect the way they cope with the challenge and,

43 thus, could be used as indicator traits to selection of disease resilience. The objectives of this study

44 were to estimate genetic parameters of feed and water intake and behavior traits for wean-to-finish

45 pigs in a natural polymicrobial disease challenge model, estimate genetic correlations of feed and

46 water intake and behavior traits with growth rate and clinical disease traits, and to develop indicator

47 traits for selection of disease resilience.

48 Results: In general, water intake traits had moderate to high estimates of heritability, especially

49 for average daily water dispensed, duration, and number of visits (0.44 to 0.58). Similar estimates

50 were observed for corresponding feed intake traits (0.35 to 0.51). Most genetic correlation

51 estimates among drinking traits were moderate to high (0.30 to 0.92) and higher than among

52 feeding traits (0 to 0.11). Compared to other water intake traits, water intake duration and number

53 of visits had relatively stronger negative genetic correlation estimates with treatment rate and

54 mortality, especially across the challenge nursery and finisher (- -0.39 and -0.45 for treatment rate;

$55-0.20$ and -0.19 for mortality).

56 Conclusion: Most water and feed intake traits under severe disease challenge had moderate to

57 high estimates of heritability, especially for feed or water intake duration and number of visits.

58 Phenotypic and genetic correlations among feed intake traits under disease were generally low but 
59 water intake traits showed high correlations with each other. Water intake duration and number of

60 visits are potential indicator traits to select for disease resilience because of their high heritability

61 and had moderate genetic correlations with treatment and mortality rates under severe disease.

63 Keywords: feeding and drinking behavior, disease resilience, genetic parameters, pigs

64

65

66

67

68

69

70

71

72

73

74

75

76

77

78

79

80

81

82 2018). Disease resilience is, however, difficult to incorporate in breeding programs because

83 nucleus breeding stock must be raised in high-health conditions, preventing the collection of 
84 disease resilience data. To collect data on disease resilience and study the genetic basis of response 85 of pigs to multiple diseases, a natural polymicrobial disease challenge model was established at a 86 research station in Quebec, Canada (Putz et al. 2019). Estimates of genetic parameters of

87 production and clinical disease data from this model were reported by Putz et al. (2019) and Cheng 88 et al. (2020).

89 Behavioral changes in eating and drinking are one of the first observable clinical signs 90 when animals are exposed to stress such as disease or extreme temperatures. Berghof et al. (2018)

91 has explored the opportunities to determine new resilience indicators based on longitudinal data.

92 Putz et a1. (2019) and Cheng et al. (2020) showed that a pigs' feeding behavior under a disease

93 challenge is genetically associated with disease resilience. A pig's drinking pattern or behaviors

94 may also change when affected by disease (Fortin et al. 2018; Kruse et al. 2011; Pedersen and

95 Madsen. 2001). The amount of water each pig drinks and drinking behaviors such as the number

96 of visits to the trough or other drinking systems, and duration of drinking on a daily basis or across

97 the test period can vary significantly with disease and stress levels, as well as with temperature,

98 humidity, and diet (Stockill, 1991). Specifically, Dybkjaer et al. (2006) reported that diarrhea in

99 young pigs could be detected about one day before the clinical signs were apparent by monitoring

100 water usage. Ahmed et al. (2015) reported that Salmonella infection in pigs resulted in reduced

101 feeding and drinking activity. Kruse et al. (2011) used the wavelet transform to analyze water

102 intake patterns to differentiate healthy and non-healthy sows. Changes in drinking behaviors in

103 diseased pigs may reflect the way they cope with the pathogen, thereby indicating the health status

104 and the level of motivation energy, which are partly influenced by the genotype of the pig (Ivoš et 105 al. 1981). 
107 intake and behavior traits for wean-to-finish pigs from a natural disease challenge model; 2)

108 estimate the genetic relationship of feed and water intake and behavior traits with growth rate and

109 clinical disease traits; 3) evaluate the usefulness of day-to-day variation and proportion of off days

110 derived from drinking behavior traits as indicators of disease resilience; and 4) develop other water

111 and feed intake behavior indicator traits to select for disease resilience. For this study, the growth,

112 feed intake, and clinical disease data analyzed by Cheng et al. (2020) were used but the focus

113 herein was on drinking behavior traits that were not analyzed previously. Results for some feed

114 intake and behavior traits from Cheng et al. (2020) are reported herein for comparison with results

115 obtained here for water intake and behavior traits.

116

117

118

119

120

121

122

123

124 du Québec (CDPQ) in Québec, Canada, and its herd veterinarian together with project 125 veterinarians.

126

\section{Data collection}

128

129

130

131

\section{MATERIALS AND METHODS}

This study was carried out in accordance with the Canadian Council on Animal Care guidelines (CCAC; https:((www.ccac.ca(en(certification(about-certification). The protocol was approved by the Protection Committee of the Centre de Recherche en Sciences Animales de Deschambault (CRSAD) and the Animal Care and Use Committee at the University of Alberta (AUP00002227). The project was fully overseen by the Centre de développement du porc$$
\text { veterinarians. }
$$

\section{Data collection}

All data and samples were collected by trained research staff from CDPQ using established natural challenge protocols, as described by Cheng et al. (2020). Data on 3,285 Large White by Landrace barrows from seven breeding companies were available. The natural challenge protocol 
132 consisted of three phases: (1) quarantine nursery (19 days on average, beginning at 3 week of age);

133 (2) challenge nursey (27 days on average); and (3) finishing phase (100 days on average). The 134 average group sizes in the three phases were 4.25, 7.16, and 10.72 pigs per pen, respectively. Pigs 135 were re-grouped when moved to the challenge nursery and to the finisher.

136 Details of the phenotypes recorded and analyzed in the challenge nursery and finisher were

137 described in Cheng et al. (2020). For health scores, treatment rates, and growth rate, data from pigs 138 that died in the finisher were included in the analyses, with imputation and expansion of treatment 139 and growth rates, as described in Cheng et al. (2020). Health scores were assigned by trained 140 personnel based on clinical signs on a 1 to 5 scale: $1=$ severe clinical signs with wasting and $5=$ 141 in perfect health, as described by Cheng et al. (2020). Treatment rate was adjusted by multiplying

142 the number of treatments a pig received in the corresponding phase by the ratio of the average 143 length of the phase and the number of days the pig spent in the phase. Mortality was recorded as $1440=$ survived and $1=$ died.

145 Individual feed intake data were recorded in the finishing barn using IVOG feeding stations 146 (Insentec, Marknesse, Netherlands) and edited using the methods of Casey et al. (2005). Individual 147 water intake data were also recorded in the finishing barn using a individual water intake recording 148 system for each pen. Designed and developed by CDPQ staff, the system allowed the recording of 149 water intake and associated data for each pig's visit. A radio frequency identification system (RFID) 150 was used for the identification of the pigs. The water delivery system includes a $3 \mathrm{~L}$ bowl that is 151 closed on 3 sides to reduce water waste, a water nipple in the bowl that can be activated by the 152 pigs, a water meter on the line that feeds the nipple, and a water level meter for the bowl. For each 153 visit, the system records the time of day, the duration, and the amount of water dispensed from the 154 nipple (dispensed) and removed from or added to the bowl. Water disappearance for each visit was 
155 calculated as water dispensed plus the change in water level in the bowl. For water and feed intake

156 traits, only data on pigs that survived to slaughter were included in analysis. For feed intake,

157 average daily feed intake, duration, and number of visits were analyzed. In total, feed intake data

158 for 2,337 pigs were included in analyses, while water intake data on 2,331 pigs were available 159 before data cleaning.

160 All animals were genotyped with the $650 \mathrm{k}$ Affymetrix Axiom Porcine Genotyping Array

161 by Delta Genomics (Edmonton AB, Canada). The 435,172 SNPs that passed quality control, as

162 described by Cheng et al. (2020), were utilized for analysis.

163

164

165

166

167

168

169

170

171 for each day and each pig as the ratio of water disappearance and intake duration. For the number

172 of visits, visits of the same pig separated by less than 10 seconds were combined as one visit and

173 a visit with zero water consumption was not counted as a visit. Because water dispensed was more

174 heritable than water disappearance ( 0.44 versus 0.34 , see later), water dispensed and dispense rate

175 were also computed for each day and each pig, i.e. ignoring data from the bowl.

176 Representative water and feed intake data for a randomly selected pig are shown in Figures

177 S1 to S4, illustrating the variability in daily water intake data, which was much larger than for feed

178 intake data, in part because of the technical difficulty of measuring water intake. To reduce the 179 effect of measurement erros, very strict editing protocols were implemented for the water intake 
data. Firstly, for each water intake trait defined above, outliers were identified on a daily basis

181 within each batch if a pig's data for that day was greater than the predicted mean +2 times the

182 predicted interquartile range (IQRP) for that day and batch. The predicted mean was based on

183 linear-quadratic regression of the water intake trait data on date by batch, as shown in Figure 1 and

184 S5, while the IQRP was based on linear-quadratic regression of the IQR by day for that batch on

185 date, as shown in Figure 1. A pig's data for a given day were removed for all water intake traits if

186 an outlier was detected for one or more traits for that day. This resulted in removal of $9 \%$ of water

187 intake days across all pigs. Secondly, because of the importance of having data at the start and end

188 of the test period, data on pigs with more than 10 missing water intake days at the start (around 70

189 to 90 days age) or at the end (around 130 to 150 days of age) of the finisher period were removed

190 by trait. This removed $32 \%$ to $41 \%$ of pigs, depending on the trait. Although removal of such a

191 large portion of data can be a concern, these editing steps increased estimates of heritability for all

192 traits by 52 to $100 \%$, suggesting that the edits removed data that contained a lot of noise.

193

194 Derivation of water intake phenotypes

195 After outlier removal, the following phenotypic random regression model was used to 196 predict the water intake trait for each pig for each day:

$$
y_{i j k}=\text { Batch }_{i}+b_{1} * A g e_{i j k}+b_{2} * A g e_{i j k}^{2}+P e n_{j}+\sum_{l=0}^{2} a_{i j k l} * A g e_{i j k}^{l}+e_{i j k}(1)
$$

198 where $y_{i j k}$ is the water intake phenotype; $B a t c h$ is a fixed batch effect $(i=1, \ldots, 50) ; A g e_{i j k}$ is

199 the entry age; $b_{1}$ and $b_{2}$ are fixed regression coefficients; $P e n_{j}$ is the random effect of finisher pen

200 within batch, with vector $\boldsymbol{P e n} \sim N\left(\mathbf{0}, \boldsymbol{I} \sigma_{P}^{2}\right)$, where $\sigma_{P}^{2}$ is the pen variance and $\boldsymbol{I}$ is the identity.

201 matrix; $\sum_{l=0}^{2} a_{i j k l} * A g e_{i j k}^{l}$ is the random regression on age for pig $k$, where $a_{i j k l}$ denotes the

202 random regression coefficients for the $k^{\text {th }}$ animal, and $l$ is the order of the polynomial $(l=0,1$, and 
203 2), with variance-covariance structure for the random regression coefficients for an individual 204 equal to $\operatorname{Var}\left[\begin{array}{l}a_{i j k 0} \\ a_{i j k 1} \\ a_{i j k 2}\end{array}\right]=\left[\begin{array}{ccc}\sigma_{0}^{2} & \sigma_{0,1} & \sigma_{0,2} \\ \sigma_{0,1} & \sigma_{1}^{2} & \sigma_{1,2} \\ \sigma_{0,2} & \sigma_{1,2} & \sigma_{2}^{2}\end{array}\right]$, where $\sigma_{0}^{2}, \sigma_{1}^{2}$, and $\sigma_{2}^{2}$ are the variances for the 205 intercept, linear, and quadratic regression coefficient, respectively, and $\sigma_{0,1}, \sigma_{0,2}$, and $\sigma_{1,2}$ are the 206 corresponding covariances; $e_{i j k}$ is the residual effect, with vector $\boldsymbol{e} \sim N\left(\mathbf{0}, \boldsymbol{I} \sigma_{e}^{2}\right)$, where $\sigma_{e}^{2}$ is the 207 residual variance, allowing for heterogeneous residual variances by age class: 53 to 82,83 to 112 , 208113 to 142,143 to 172 , and greater than 172 days.

209 Average daily water intake phenotypes were computed based on the predicted values from 210 the random regression model and included average daily water dispensed (ADWD), average daily 211 water disappearance (ADWI), average daily water intake duration (WIDUR), average daily 212 number of water intake visits (WInVisits), average daily water dispensed rate (WDRT), and 213 average daily water disappearance rate (WIRT). Day-to-day variation in phenotypes was computed

214 for each pig as the mean square root of deviations of observed from predicted values from the 215 random regression model (Figure 2) and included day-to-day variation in water dispensed 216 (VAR $\left.\mathrm{WD}_{\mathrm{WD}}\right)$, water disappearance $\left(\mathrm{VAR}_{\mathrm{WI}}\right)$, water intake duration (VAR $\left.\mathrm{WIDUR}_{\mathrm{R}}\right)$, number of water 217 intake visits (VARWInVisits), water dispense rate (VARWDRT), and water disappearance rate 218 (VARwIRT). The coefficient of variation for day-to-day variation phenotypes were calculated as 219 the square root of the mean of the day-to-day variation of the water intake trait divided by the mean 220 of corresponding average daily water intake trait across pigs, e.g. $\sqrt{\mathrm{VAR}_{W I}} / \mathrm{ADWI}$. The proportion 221 of off-water days for each water intake trait was computed as the number of off-water days divided 222 by total number of water intake days and included the proportion of off-water days based on water 223 dispensed $\left(\mathrm{OFF}_{\mathrm{WD}}\right)$, water disappearance $\left(\mathrm{OFF}_{\mathrm{WI}}\right)$, water intake duration (OFF $\left.\mathrm{WIDUR}\right)$, number of 224 water intake visits $\left(\mathrm{OFF}_{\mathrm{WInVisits}}\right)$, water dispense rate $\left(\mathrm{OFF}_{\mathrm{WDRT}}\right)$, and water disappearance rate 
$225\left(\mathrm{OFF}_{\mathrm{WIRT}}\right)$. A day for a pig was considered an off-water day if the residual for that day from the

226 random regression model was less than the IQRP of residuals (Figure 2), where the IQRP was

227 obtained for each water intake day and each batch using linear quadratic regression of the observed

228 IQR of residuals on water intake date. Pigs with more than $30 \%$ off-water days for a given trait

229 were removed because their data likely reflects a malfunction of the water drinking system rather

230 than poor resilience. This resulted in removal of $0.2,5,18,18,5$, and $7 \%$ of pigs for OFFWInVisits,

231 OFFWIDUR, OFFWI, OFFWD, OFFWIRT, and OFFWDRT, respectively.

232 To determine whether off-water days coincided with off-feed days, a pig's off-water days

233 were compared with that pig's off-feed days throughout the finishing period for days for which a

234 pig had both feed and water intake. In contrast to off-water intake days, off-feed intake days were

235 identified based on quantile regression, as described by Putz et al. (2019). To explore the

236 relationship between off days and health treatments, overlaps of off-water or off-feed days with

237 days in which the pig received treatment were also investigated. To allow for some difference in

238 the timing of off-water, off-feed, and treatment days, a 7-day rolling window was also used to

239 identify the coincident off-feed, off-water, and treatment days. For example, off-water and off-

240 feed events were considered to overlap for a 7-day window if that window included at least one

241 off-water and one off-feed day. A similar strategy was used for overlaps with treatments. Chi-

242 square tests were used to determine whether off-water, off-feed, and treatment events coincided

243 significantly more than expected. The expected number of overlapping off-feed and off-water days

244 was computed as the products of the proportion of off-feed days, the proportion of off-water days,

245 and the total number of days with feed or water intake data $(92,682)$. The expected coincident off-

246 feed or off-water days with treatment were computed as the products of the proportion of off-feed

247 or off-water days, the proportion of treatment days, and the total number days with feed or water 
248 intake data. When using 7-day windows, proportions and numbers of days were replaced by

249 proportions and numbers of windows with at least one off-water or off-feed or treatment day.

\section{Variance component estimation}

Variance components were estimated by genomic best linear unbiased prediction (GBLUP)

254 using ASReml 4.0 (Gilmour et al, 2015). The following general model was used in single-trait and 255 bivariate analyses to estimate variance components and genetic correlations:

$$
y_{i j k}=\text { Batch }_{i}+A g e_{i j k}+\text { Pen }_{j}+u_{i j k}+\text { litter }_{i j k}+e_{i j k}
$$

257 where $y_{i j k}$ is the trait; $B a t c h$ is a fixed batch effect $(\mathrm{i}=1, \ldots, 50) ; A g e_{i j k}$ is the covariate of age

258 when the pig entered the quarantine nursery; $P e n_{j}$ is the random effect of pen by batch

259 corresponding the different phases, with vector $\boldsymbol{P e n} \sim \mathrm{N}\left(\mathbf{0}, \boldsymbol{I} \sigma_{P}^{2}\right)$, where $\sigma_{P}^{2}$ is the pen variance;

$260 u_{i j k}$ is the random additive genetic effect, with the vector $\boldsymbol{u} \sim \mathrm{N}\left(\mathbf{0}, \boldsymbol{G} \sigma_{A}^{2}\right)$, where $\boldsymbol{G}$ is the genomic

261 relationship matrix and $\sigma_{A}^{2}$ is the additive genetic variance; litter $_{i j k}$ is the litter environmental

262 effect, with vector litter $\sim \mathrm{N}\left(\mathbf{0}, \mathbf{I} \sigma_{l}^{2}\right)$, where $\sigma_{l}^{2}$ is the litter environmental variance; $e_{i j k}$ is the

263 residual effect, with vector $\boldsymbol{e} \sim \mathrm{N}\left(\mathbf{0}, \boldsymbol{I} \sigma_{e}^{2}\right)$, where $\sigma_{e}^{2}$ is the residual variance. The genomic

264 relationship matrix, $\boldsymbol{G}$, was created separately for each company using the software preGSf90

265 (Misztal et al., 2002) based on method one of VanRaden (2008). Then, the seven $\boldsymbol{G}$ matrices were

266 combined into one $\boldsymbol{G}$ matrix, with genetic relationships between companies set to zero, such that

267 the analyses focused on pooled within-company variances. Water-to-feed and feed-to-water ratio

268 traits were also analyzed as conditional traits by fitting the corresponding feed or water intake

269 phenotype of that animal as a covariate. For example, for analysis of ADFI conditional on ADWI,

270 ADWI was fitted as a covariate in the model for analysis of ADFI. 


\section{Phenotypic data}

\section{RESULTS}

Table 1 shows phenotypic means (SD) for all water intake, feed intake, and behavior traits,

275 and the numbers of records retained for analysis. Comparing feed with water intake behaviors,

276 pigs spent more than three times as much time eating than drinking on a daily basis but pigs made

277 nearly twice as many visits to the drinker than to the feeder per day. As a result, pigs spent six

278 times more time eating than drinking per visit. Day-to-day variation in feed intake duration was

279 nearly three times greater than day-to-day variation in water intake duration, while day-to-day

280 variation for the number of feed intake visits was less than for the number of water intake visits.

281 Day-to-day variation in daily feed intake and in feed intake rate was lower than for water

282 disappearance and water disappearance rate. However, the coefficients of day-to-day variation

283 were greater for feed intake, number of visits, and feed intake rate than for the corresponding water

284 intake traits. The proportions of off-water or off-feed intake days were small but twice as large for

285 water intake traits than for feed intake traits, noting that different methods were used to identify

286 off-water versus off-feed intake days.

287 Using only data on days for which a pig had both feed and water intake data, $54 \%$ of pigs

288 had at least one off-feed day, $96 \%$ of pigs had at least one off-water day, and $41 \%$ of pigs received

289 at least one treatment. On a daily basis, $2 \%$ of days were classified as off-feed, $12 \%$ as off-water,

290 and $1 \%$ as treatment. Results for the overlap between off-days and treatment rate are in Table 2.

291 Co-incidences of off-feed and off-water days and treatment days were all significantly $(\mathrm{P}<0.0001)$

292 greater than expected based on a Chi-square test, by a factor that ranged from 1.4 to 12.2 (Table

293 2), both on a daily basis and on a 7-day rolling window basis. On the basis of the 7-day rolling 
294 window, 10, 41, and 6\% of windows had at least one off-feed, off-water, or treatment day, 295 respectively.

\section{Heritabilities of feed and water intak and behavior traits}

Table 1 shows estimates of heritability and litter effects for the water intake, feed intake, 299 and behavior traits. Water intake traits in general had moderate to high estimates of heritability, 300 especially average daily water dispensed, duration, and number of visits (0.44 to 0.58). Similar 301 estimates were observed for corresponding feed intake traits (0.35 to 0.51$)$. Note that water 302 dispensed (ADWD) and dispensed rate (WDRT) had higher estimates of heritability than water 303 disappearance (ADWI) and disappearance rate (WIRT), respectively. Interestingly, estimates of 304 heritability increased for all water intake traits (to 0.42 to 0.60 ) when the corresponding feed intake 305 trait was fitted as a covariate. Similar increases were observed for feed intake and duration (to 0.42 306 and 0.55 , respectively) when fitting the corresponding water intake trait as a covariate. Day-to307 day variation in water intake traits had moderate estimates of heritability (0.22 to 0.38$)$, while 308 corresponding feed intake traits had a wider range of estimates (0 to 0.47 ). Day-to-day variation

309 in the number of feed intake visits had the highest estimate of heritability (0.47), while day-to-day 310 variation in feed intake and intake rate had low estimates of heritability ( 0.08 and 0 , respectively).

311 The proportion of off-days had low to moderate estimates of heritability for both water intake and 312 feed intake traits ( 0.15 to 0.31 and 0.10 to 0.26 , respectively). Litter effects were low for all traits, 313 ranging from 0 to 0.13 . 
Table 3 shows estimates of genetic and phenotypic correlations among water and feed

317 intake and behavior traits. Most of the genetic correlation estimates among water intake traits were

318 moderate to high ( 0.30 to 0.92$)$, except for water disappearance rate with water intake duration

319 and number of visits (0.10 and -0.07). Note that water dispensed was highly correlated with water

320 disappearance, both genetically and phenotypically (0.84 and 0.79$)$. In contrast, most genetic

321 correlations among feed intake traits were estimated to be low (0 to 0.11$)$, except for feed intake

322 rate with average daily feed intake (0.44). The majority of phenotypic correlations among water

323 intake traits were also moderate to high (0.31 to 0.90$)$, except for water intake duration with intake

324 rate (0.09) and number of water intake visits with dispense rate and intake rate $(0.09$ and -0.08 ,

325 respectively). Phenotypic correlations among feeding traits were low, except for feed intake rate

326 with average daily feed intake and duration (0.51 and -0.70 , respectively).

327 Table 4 shows estimates of phenotypic and genetic correlations between corresponding

328 water and feed intake and behavior traits. Surprisingly, the water intake traits were not highly

329 correlated with feed intake traits, either genetically $(0.08$ to 0.36$)$ or phenotypically $(0.13$ to 0.39$)$.

330 The same was true for day-to-day variation and off-days traits.

331

332 Correlations of water and feed intake and behavior traits with growth traits

333 Estimates of genetic and phenotypic correlations of water and feed intake and behavior

334 traits with growth rate in the three phases (quarantine nursery, challenge nursery, and finisher) are

335 shown in Table 5. Water intake traits in general had low positive genetic correlation estimates with

336 growth rate in the quarantine nursery but moderate positive genetic correlation estimates with

337 growth rate in the challenge nursey and finisher. This means that, genetically, pigs that had higher

338 growth rate under challenge, drank more water in the finisher, spent more time drinking, paid more 
339 visits to the drinker, and drank faster. Average daily feed intake and feed intake rate had moderate-

340 to-high positive genetic correlation estimates with growth rate in the challenge nursey and finisher

341 but lower genetic correlations with growth rate in quarantine nursery. Feed intake duration and

342 number of visits, however, had very low and even negative genetic correlation estimates with

343 growth rate in the different phases. This indicates that, genetically, pigs that had higher growth

344 rate under challenge, ate more feed and ate faster in the finisher. Fitting feed or water intake traits

345 as covariates for water and feed intake traits had minimal effects on the correlation estimates for

346 growth rate, except for genetic correlations of ADWI and ADWD with finisher ADG, which

347 decreased to near zero.

348 Most day-to-day variation traits for water intake traits had low positive genetic correlation

349 estimates with growth rate in the quarantine and challenge nursery. Estimates of correlations with

350 growth rate in finisher were stronger for day-to-day variation in water disappearance, dispensed,

351 and duration $(0.21,0.20$, and 0.27 , respectively) than for day-to-day variation in number of visits,

352 disappearance rate, and dispensed rate. Day-to-day variation in feed intake traits had stronger

353 genetic correlation estimates with growth rate than day-to-day variation in the corresponding water

354 intake traits. Day-to-day variation in daily feed intake had high positive genetic correlation

355 estimates with growth rate in the quarantine and challenge nursery (0.55 and 0.65) and a moderate

356 genetic correlation estimate with growth rate in the finisher (0.30). Day-to-day variation in feed

357 intake duration and number of visits, however, had moderate negative genetic correlation estimates

358 with growth rate in the challenge nursery and finisher. Day-to-day varitation in feed intake rate

359 had very low estimates of heritability and, thus, had genetic correlation estimates with high

360 standard errors. For the proportion of off-water or -feed days, off-water traits generally had lower

361 genetic correlation estimates with growth rate than off-feed traits. Off-days for feed intake and 
362 intake rate had moderate-to-high negative genetic correlation estimates with growth rate in the 363 challenge nursery and finisher (-0.35 to -0.80). Off-days for feed intake duration also had a high

364 negative genetic correlation estimate with growth rate in the finisher. Estimates of phenotypic 365 correlations in general were of a similar magnitude as genetic correlation estimates for off-days 366 for all water and feed intake traits.

\section{Correlations of water and feed intake and behavior traits with clinical disease traits}

Table 6 shows estimates of phenotypic correlations of water and feed intake and behaviors

370 with health scores and with treatment and mortality rates in the different phases. Phenotypic

371 correlations with mortality rates were not computed because only pigs that survived were included

372 for the water and feed intake trait analyses. Water intake traits generally had low phenotypic

373 correlation estimates with health scores and treatment rates. Average daily feed intake and intake

374 rate, however, had moderately high phenotypic correlation estimates with health score in the

375 finisher $(0.39$ and 0.24$)$ and with treatment rates (-0.18 to -0.30 for feed intake and -0.09 to -0.17

376 for intake rate). Fitting feed or water intake traits as covariates for water and feed intake traits did

377 not substantially change their correlation estimates with health scores and treatment rates, except

378 for ADWI and ADWD, for which phenotypic correlations decreased. Similar trends were found

379 for day-to-day variation and off-days for water intake traits, which generally had low phenotypic

380 correlation estimates with health scores and treatment rates. By contrast, day-to-day variation and

381 off-days for feed intake and duration traits tended to have stronger phenotypic correlation estimates

382 with health score in the finisher and with treatment rates. The proportion of off-feed days for intake

383 rate also had strong phenotypic correlation estimates with health scores and treatment rates. 
385 disease traits are shown in Table 7. Treatment rate in the finisher had a zero estimate of heritability 386 and was, thus, excluded from these analyses. Interestingly, water intake duration and number of 387 visits had relatively stronger negative genetic correlation estimates with treatment and mortality 388 rates than other traits, especially across the challenge nursery and finisher $(-0.39$ and -0.45 for 389 treatment rate; -0.20 and -0.19 for mortality). This implies that, genetically, pigs that were more 390 likely to die or that received more treatments spent less time drinking and paid fewer visits to 391 drinker. Average daily water disappearance and dispensed were genetically positively correlated 392 with health scores and negatively correlated with treatment and mortality rates, but these 393 correlation estimates were not strong in general. Interestingly, water disappearance rate had 394 moderate genetic correlation estimates with health scores in the challenge nursery and finisher 395 (0.41 and 0.36), which means that pigs with higher health scores, genetically had a higher water 396 disappearance rate. For feeding traits, feed intake duration and number of visits had moderately 397 negative genetic correlation estimates with mortality in the finisher and across the challenge 398 nursery and finisher (-0.23 to -0.43$)$, which means that, genetically, pigs that were more likely to 399 die spent less time eating and paid fewer visits to feeder. Average daily feed intake had moderate 400 genetic correlation estimates with treatment rate $(-0.25$ to -0.33$)$ and health score in the finisher 401 (0.31). Fitting feed or water intake traits as covariates for water and feed intake traits in general 402 had minimal effect on estimates of genetic correlations with clinical traits, except for ADWI and 403 ADWD with health score in the finisher and with treatment rate across the challenge nursery and 404 finisher, for which adding the covariate decreased the genetic correlation estimates.

405 Day-to-day variation in water intake traits in general had low to moderate genetic 406 correlations with health scores and with treatment and mortality rates (Table 7). However, these 
407 correlation estimates, e.g., of day-to-day variation in water intake duration with treatment rate (-

4080.23 to -0.43 ) had unexpected directions because pigs that are less resilient were expected to have

409 higher day-to-day variation in water intake traits. Day-to-day variation in feed intake traits

410 generally had moderate to high genetic correlation estimates with resilience traits and in the

411 expected direction.

412 Most off-days traits for water intake traits had low genetic correlation estimates with health

413 scores and with treatment and mortality rates, except for off-days for water dispensed with

414 mortality in the finisher and across the nursery and finisher (-0.46 and -0.37) and for off-days for

415 water intake duration with treatment rate across the nursery and finisher (-0.46), but again in the

416 unexpected direction. On the other hand, the proportion of off-days for for feed intake had strong

417 genetic correlation estimates with health score in the finisher $(-0.45)$, with treatment rate in the

418 finisher and across the nursery and finisher (0.38 and 0.51), and with mortality across the nursery

419 and finisher (0.48), all in the expected direction. Off-days for number of feed intake visits had

420 moderate positive genetic correlation estimates with mortality in the finisher and across the nursery

421 and finisher (0.32 and 0.34). Off-feed days for feed intake rate had moderate to high genetic

422 correlation estimates with health score in the finisher $(-0.36)$, with treatment rate in the finisher

423 and across the nursery and finisher (0.28 and 0.48$)$, and with mortality in the challenge nursery

424 (0.60), all in the expected direction.

425

426

\section{DISCUSSION}

427 Although several studies have explored the impact of water intake on pig production

428 performance and health (Fortin et al. 2018; Kruse et al. 2011; Pedersen and Madsen, 2001), very

429 few studies have addressed the effect of health status on individual water intake and drinking 
430 behaviors (Ahmed at el. 2015). To our knowledge, this is the first study to report genetic

431 parameters of individual water intake and drinking behaviors, especially under a disease challenge.

432 In this study, we were also able to combine feed intake and water intake data and explore the 433 relationship between feeding and drinking behaviors under a severe disease challenge and the 434 relationship of these traits with disease resilience. Water intake is difficult to measure because pigs 435 like to play with water, especially in hot weather, which makes individual water intake data noisier 436 than individual feed intake data. To address this, we developed stringent methods for quality 437 control and editing of the water intake data prior to analysis. Although this removed a large 438 proportion of the data (9\% of water intake days and 32 to $41 \%$ of pigs, depending on the trait), we 439 believe these data processing steps were critical. In fact, estimates of heritability for the water 440 intake traits increased by 50 to $100 \%$ following quality control editing. The resulting data and 441 analyses provide novel insights into the phenotypic and genetic relationships among feed and water 442 intake behavior traits under disease, as well as their relationships with disease resilience.

444 Genetic parameters of water and feed intake and behavior traits under a severe disease 445 challenge

446 1. Heritability

447 Many estimates of genetic parameters of feeding behaviors in generally healthy pigs have 448 been reported (Labroue et al,1997; Hall et al, 1999; Schulze et al, 2003; McSweeny et al, 2003; 449 Ding et al, 2018). Reported estimates of heritability for feed intake duration per day, number of 450 visits per day, and feed intake rate range from 0.31 to 0.46 , from 0.29 to 0.43 , and from 0.41 to 4510.50 , respectively. These estimates were in general slightly lower than the estimates reported in 452 this study (Table 1), where pigs were under a severe disease challenge. However, to our knowledge, 
453 no studies have reported genetic parameters of water intake and drinking behaviors. Nevertheless,

454 the water intake traits had estimates of heritability that were of a similar magnitude as those for

455 feed intake traits. Water intake duration and number of visits per day were the most heritable

456 among the water intake traits and were more heritable than the corresponding feed intake traits.

457 Additionally, both feed and water intake duration and number of visits per day were more heritable

458 than daily feed intake and daily water disappearance or dispensed. It should be noted that daily

459 water dispensed was more heritable than water disappearance. The coefficient of variation was

460 much higher for water dispensed than water disappearance (Table 1), which shows that water

461 dispensed had higher variability. The same holds true for rates of water dispensed and

462 disappearance. However, water dispensed and disappearance were strongly correlated with each

463 other both phenotypically (0.79) and genetically (0.84), which was as expected, as both traits are

464 a combination of water consumption, wastage, and playing behavior.

465 Putz et al. (2019) and Cheng et al. (2020) reported low to moderate estimates of heritability

466 for day-to-day variation and the proportion of off-days derived from feed intake and duration.

467 Similar results were also reported here because they were based on mostly the same feed intake

468 data (Table 1). Day-to-day variation in the number of feed intake visits, which was not investigated

469 in these previous studies, was found to be highly heritable. In general, both day-to-day variation

470 and the proportion of off-days for water intake traits were low to moderately heritable.

471

\section{2. Relationships between water and feed intake traits}

473 In general, estimates of phenotypic and genetic correlations among feed intake traits were

474 very low (Table 3$)$. Estimates of the phenotypic (0.15) and genetic (0.10) correlation between daily

475 feed intake and duration were much lower than the range of estimates reported in the literature 
476 (0.17 to 0.88 and 0.14 to 0.97 , respectively) (Labroue et al,1997; Schulze et al, 2003; McSweeny

477 et al, 2003; Ding et al, 2018). Cheng et al. (2020) suggested that the low correlations in these data

478 were probably because feed intake and duration were differently affected by the disease challenge.

479 The average number of daily feed intake visits also had low phenotypic (-0.01) and genetic $(0.11)$

480 correlations with daily feed intake and were also quite different than estimates reported in the

481 literature (-0.4 to -0.09 and -0.35 to 0.02 , respectively) (Labroue et al,1997; Schulze et al, 2003;

482 McSweeny et al, 2003; Ding et al, 2018). The average number of daily feed intake visits also had

483 low phenotypic and genetic correlations with daily feed intake duration $(0.11$ and 0.08 ,

484 respectively) but these estimates were in the range of estimates reported in the literature (-0.12 to

4850.15 and -0.21 to 0.38 , respectively, Labroue et al,1997; Schulze et al, 2003; McSweeny et al,

486 2003; Ding et al, 2018). It is noteworthy that the range of correlation estimates in the literary is

487 large, which could result from differences in feeding systems, breeds, environments, analytical

488 models, health status, etc. The low correlations in our data may also be because the different feed 489 intake traits were differentially affected by disease challenge, as is evident from the large 490 coefficients of day-to-day variation in feed intake, duration, number of visits, and intake rate $(0.67$,

$4910.06,0.14$, and 2.5 , respectively, Table 1$)$. Feed intake rate had moderate positive phenotypic $(0.51)$

492 and genetic (0.44) correlation estimates with daily feed intake in our data (Table 3), as well as

493 strong negative phenotypic (-0.70) but very low genetic (0) correlations with duration, and very

494 low phenotypic (0.10) and genetic (0) correlations with number of feed intake visits. Except for

495 the low genetic correlation of feed intake rate with feed intake duration, these estimates were in

496 general consistent with literature estimates ( 0.41 to 0.42 for phenotypic correlations and 0.20 to

4970.49 for genetic correlations with daily feed intake; -0.76 to -0.70 for phenotypic correlations and

$498-0.86$ to -0.78 for genetic correlations with feed intake duration; -0.15 to -0.10 for phenotypic 
499 correlations and -0.22 to -0.12 for genetic correlations with number of feed intake visits) (Labroue 500 et al,1997; Schulze et al, 2003). Most estimates of phenotypic and genetic correlations among 501 water intake traits were much higher than corresponding estimates among feed intake traits (Table

502 3). Coefficients of day-to-day variation (Table 1) were more consistent across water intake than 503 across feed intake traits ( 0.09 to 1.08 versus 0.06 to 2.50 , respectively, Table 1$)$.

504 To our knowledge, no studies have reported on genetic parameters of water intake traits 505 but some studies have explored phenotypic relationships among water intake traits. Using data 506 from cameras, Kashiha et al. (2013) found that water usage was highly related with the duration 507 of drink nipple visits $\left(\mathrm{R}^{2}=0.92\right)$. Maselyne et al. (2015), using an RFID drinking system, found 508 similar magnitudes of the relationships of water usage with the number of water intake visits $\left(\mathrm{R}^{2}\right.$ $509=0.69$ to 0.75$)$ and duration $\left(\mathrm{R}^{2}=0.65\right.$ to 0.71$)$. All these studies were, however, conducting using 510 generally healthy pigs.

$511 \quad$ Phenotypic and genetic correlations between corresponding feed and water intake traits 512 were generally low (Table 4). This contradicts previous studies that suggest a high phenotypic 513 relationship between feed and water intake traits (Huynh et al. 2005; Ahmed et al. 2015). However, 514 these studies were conducted under conditions that were very different from our study, where pigs 515 were under severe disease challenge, which may disproportionately affect feed and water intake, 516 resulting in a reduction in the relationships between feed and water intake traits. In the present 517 study, pigs were severely challenged with multiple viral and bacterial pathogens and resulted in 518 chronic disease, particularly of the respiratory tract. Additionally, other factors, such as social 519 stress and vaccination, could affect the pattern of water intake and feed intake.

\section{Relationship of water and feed intake traits with growth rate}


Average daily feed intake was strongly correlated with average daily gain (ADG) in the

523 finisher, both phenotypically (0.86) and genetically (0.84), which is consistent with the literature

524 ( 0.67 to 0.73 for phenotypic correlations and 0.77 to 0.87 for for genetic correlations) (Labroue et

525 al,1997; McSweeny et al, 2003; Young et al. 2012), although pigs were under a severe disease

526 challenge in this study. Feed intake rate was also moderately correlated with ADG in the finisher,

527 both phenotypically (0.34) and genetically (0.42) and consistent with estimates in the same

528 literature $(0.25$ to 0.28 for phenotypic correlations and 0.17 to 0.48 for genetic correlations)

529 (Labroue et al,1997; McSweeny et al, 2003; Young et al. 2012). Interestingly, daily feed intake

530 and intake rate (recorded in the finisher) were also moderately correlated with ADG in the

531 challenge nursery, both phenotypically (0.40 to 0.42$)$ and genetically (0.34 to 0.49), although ADG

532 in the challenge nursey was weakly correlated with ADG in the finisher, both phenotypically $(0.20)$

533 and genetically (0.15) (Cheng et al. 2020). This means pigs that grew faster in the challenge

534 nursery ate more feed and ate faster in the finisher. Feed intake duration and number of visits,

535 however, had very low phenotypic (0.16 and -0.03 , respectively) and genetic (0.09 and -0.01 ,

536 respectively) correlations with $\mathrm{ADG}$ in the finisher but in the range of estimates reported in the

537 literature ( 0.13 to 0.64 for phenotypic correlations and 0.02 to 0.69 for genetic correlations with

538 duration; -0.09 to 0.01 for phenotypic correlations and -0.22 to -0.03 for genetic correlations with

539 number of visits) (Labroue et al,1997; McSweeny et al, 2003; Young et al. 2012). Water intake

540 traits in general had low correlations with ADG in the nursery and the finisher, both phenotypically

541 (-0.02 to 0.34$)$ and genetically (-0.02 to 0.39$)$. Very few studies have reported relationships of

542 water intake with growth rate in pigs but Brew et al. (2011) reported a very low phenotypic

543 relationship $\left(\mathrm{R}^{2}=0.005\right)$ of water intake with ADG in generally healthy growing beef cattle. 
545 phenotypic and genetic correlations with ADG in the nursery and the finisher (Table 5). In contrast,

546 the proportion of off-days for feed intake, duration, and intake rate had moderate to strong negative

547 phenotypic and genetic correlations with ADG in the finisher (-0.42 to -0.80$)$, as was already

548 established based on these data by Cheng et al. (2020).

\section{Relationship of water and feed intake traits with clinical disease traits}

There was clear evidence that off-feed, off-water, and treatment events coincided more

552 often than expected by a factor of 1.42 to 12.22 (Table 2). This suggests that the feeding and

553 drinking behaviors are related to the health status of pigs. However, feed and water intake traits in

554 general had low phenotypic correlations with clinical disease traits (Table 6), except for the

555 proportion of off-feed intake days with health score in the finisher $(-0.35)$ and with treatment rate

556 across the nursery and finisher ( 0.25 to 0.38 ). This could be because in the entire finishing period,

557 only $41 \%$ of pigs received treatment and there were not many days pigs received medical

558 treatments. Putz et al. (2019) and Cheng et al. (2020) demonstrated that day-to-day variation and

559 the proportion of off-days for feed intake and duration were highly associated with disease

560 resilience, using the same feed intake data as used herein. Water intake traits, however, consistently

561 had low phenotypic correlations with clinical traits.

562 Estimates of genetic correlations of feed and water intake traits with clinical traits were in

563 general much stronger than the corresponding phenotypic correlations (Table 6 and 7). Specifically,

564 as already established by Cheng et al. (2020) for these data, average daily feed intake had

565 moderately high genetic correlation estimates with health score in the finisher and with treatment

566 rates but a low estimate with mortality. In contrast, feed intake duration and number of visits had 
567 moderately high genetic correlation estimates with mortality but low estimates with health scores

568 and treatment rates. This indicates that genetically, mortality was more associated with feed intake

569 duration and number of visits, while treatment rates and health scores were more related with feed

570 intake. Water dispensed, duration, and number of visits generally had moderately high genetic

571 correlation estimates with treatment rates and mortality. It is noteworthy that water dispensed had

572 stronger genetic correlation estimates with treatment rates and mortality than water disappearance

573 did, which means that, genetically, water dispensed was more affected by disease than water

574 disappearance.

575 Day-to-day variation in feed intake had moderate to high genetic correlation estimates with

576 mortality across the nursery and finisher (Table 6), which was already reported for these data in

577 Putz et al. (2019) and Cheng et al. (2020). Day-to-day variation for feed intake duration and

578 number of visits, on the other hand, had moderately high genetic correlation estimates with health

579 scores, which were not reported previously. These moderate to high genetic correlation estimates

580 were in the expected direction (negative with health score and positive with mortality), which

581 suggests that these traits are potential indicator traits to select for disease resilience. Unfortunately,

582 although day-to-day variation for water intake duration and number of visits had moderately high

583 genetic correlation estimates with treatment rates, the negative sign of these estimates was opposite

584 to expections. This maybe because healthy pigs are more active, developing thirst, or play with the

585 drinker more than sick pigs. The proportion of off-days for feed intake had moderately high genetic

586 correlation estimates with health score in the finisher, treatment rates, and mortality, and in these

587 estimates were in the expected directions. Proportions of off-days for feed intake duration and

588 number of visits, however, only had moderately genetic correlation estimates with mortality.

589 Interestingly, the proportion of off-days for feed intake rate was estimated to be moderately 
590 genetically correlated with health score in the finisher and with treatment rates. The proportion of 591 off-days for some drinking traits was also estimated to be moderately genetically correlated with

592 clinical traits but, again, the signs of these estimates were opposite to expectations. Generally 593 speaking, there were very few feed or water intake traits that showed consistent moderate or strong 594 genetic correlations with all clinical traits. This is somewhat unexpected because strong genetic 595 correlations were reported among these three clinical traits (Cheng et al. 2020).

\section{Resilience indicator traits based on feed and water intake traits}

598 Clinical disease traits such as treatment and mortality rates have been shown to have low 599 heritability, both in the data used here (Cheng et al. 2020) and in the literature (Dufrasne et al. 600 2014; Guy et al. 2018) and are thus, difficult to improve by direct selection. Therefore, an 601 important aim of this study was to find indicator traits of disease resilience that can be selected on 602 instead. To be a useful indicator trait for indirect selection for disease resilience, it must be 603 moderately to highly heritable and have a high genetic correlation with disease resilience. Based 604 on the same data as used here, Putz et al. (2019) and Cheng et al. (2020) suggested that day-to-day 605 variation and the proportion of off-days for feed intake and duration had great potential as 606 indicators because of their moderately high heritabilities and strong genetic correlations with 607 treatment and mortality rates. In the present study, additional feed and water intake traits were 608 investiged and some showed great potential as well. The number of feed intake visits was highly 609 heritable (0.51) and moderately genetically correlated with mortality in the finisher and, thus, 610 could be used as an indicator trait to select against mortality under disease. Water intake duration 611 and number of visits also had high estimates of heritability ( 0.54 and 0.58 , respectively) and 612 moderate genetic correlations with treatment and mortality rates, especially for treatment rate 
613 across the nursery and finisher (-0.39 to -0.45). Hence, these two water intake traits also have great

614 potential as indicator traits to select for disease resilience. The water-to-feed ratio traits, obtained

615 by fitting the corresponding feed intake traits as covariates when analyzing water intake, are also

616 promising because of their even higher estimates of heritability (0.56 to 0.60$)$ and similar

617 magnitudes of genetic correlations with clinical traits as the non-ratio water intake traits.

618 Electronic feeders have been implemented in most nucleus breeding programs and 619 Harlizius et al. (2020) has proved that the variation of individual feed intake collected form the 620 nucleus farm could be used to improve finisher survival rate. Electronic feeders could also be used

621 in commercial farms to provide the data needed to implement these indicator traits. With the

622 availability of new technologies such as 3D cameras, thermal imaging, and sensors, recording

623 feeding and drinking behavior traits on commercial farms can be implemented. Incorporation of

624 these indicator traits into breeding programs could result in genetic improvement of resilience to

625 disease. To achieve this goal, a specialized disease challenge facility or one or more commercial 626 farms with severe disease issues could be used for collection of these resilience indicator traits and

627 connected to selection candidates in the nucleus using genomic prediction.

\section{8}

629

\section{CONCLUSIONS}

630 Water and feed intake traits under a severe polymicrobial disease challenge in general had 631 high estimates of heritability, especially for duration and number of visits for both feed and water 632 intake. Phenotypic and genetic correlations among feed intake traits were generally low under the 633 disease challenge but water intake traits showed high correlations amongst each other. 634 Corresponding feed and water intake traits were not strongly correlated with each other under the 635 disease challenge conditions. Water intake traits generally had low genetic correlations with 
636 growth rate under challenge but some feed intake traits such as daily feed intake and intake rate

637 had moderate to strong genetic correlations with growth rate in the finisher. Day-to-day variation

638 and the proportion of off-days for water intake traits were not highly genetically correlated with

639 treatment and mortality rates, in contrast to the corresponding feed intake traits. However, water

640 intake duration and number of visits were highly heritable and had moderately high genetic

641 correlations with treatment and mortality rates and, thus, are potential indicator traits to select for

642 disease resilience. Especially promising is the number of water intake visits, which had a high

643 heritability of 0.58 and moderately high genetic correlations of -0.45 with treatment rate and -0.19

644 with mortality.

645

\section{List of Abbreviations}

647 ADFI, average daily feed intake

648 ADG, average daily gain

649 ADWI, average daily water disappearance

650 ADWD, average daily water dispensed

651 ADWI|ADFI, ratio trait by fitting ADFI as covariate for ADWI

652 ADWD|ADFI, ratio trait by fitting ADFI as covariate for ADWD

653 ADFI|ADWI, ratio trait by fitting ADWI as covariate for ADFI

$654 \mathrm{ADFI} \mid \mathrm{ADWD}$, ratio trait by fitting ADWD as covariate for ADFI

$655 \mathrm{CV}$, coefficient of variation

656 FIDUR, average daily feed intake duration

657 FInVisits, average number of daily feed intake visits

658 FIRT: average daily feed intake rate 
659 FIDUR/WIDUR, ratio trait by fitting WIDUR as covariate for FIDUR

660 FInVisits|WInVisits, ratio trait by fitting WInVisits as covariate for FInVisits

661 FinADG, average daily gain in finisher

662 FinMOR, mortality rate for pigs in finisher

663 FinTRT, number of health treatments per pig in finisher

664 GBLUP, genomic best linear unbiased prediction

665 IQR, interquartile range

666 IQRP, predicted IQR

$667 \mathrm{OFF}_{\text {FIDUR, }}$, proportion of off-feed days for feed intake duration

$668 \mathrm{OFF}_{\mathrm{FI}}$, proportion of off-feed days for feed intake

$669 \mathrm{OFF}_{\text {FInVisits, }}$, proportion of off-feed days for number of feed intake visits

$670 \mathrm{OFF}_{\mathrm{FIRT}}$, proportion of off-feed days for feed intake rate

$671 \mathrm{OFF}_{\mathrm{WI}}$, proportion of off-water days for water disappearance

$672 \mathrm{OFF}_{\mathrm{WD}}$, proportion of off-water days for water dispensed

$673 \mathrm{OFF}_{\text {WIDUR, }}$, proportion of off-water days for water intake duration

$674 \mathrm{OFF}_{\mathrm{WInV} V \text { isits, }}$ proportion of off-water days for water intake visits

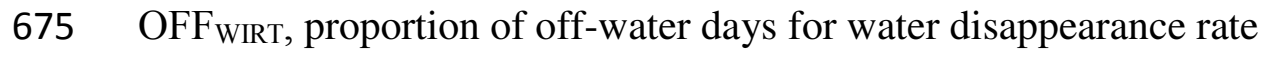

$676 \mathrm{OFF}_{\text {WDRT, }}$ proportion of off-water days for water dispense rate

677 RFID, radio frequency identification system

678 SNP, single nucleotide polymorphism

679 VAR $_{\text {FIDUR, day-to-day variation in feed intake duration }}$

$680 \mathrm{VAR}_{\mathrm{FI}}$, day-to-day variation in feed intake

$681 \mathrm{VAR}_{\mathrm{FInVisits}}$, day-to-day variation in number of feed intake visits 
682 VAR $_{\text {FIRT, day-to-day variation in feed intake rate }}$

$683 \mathrm{VAR}_{\mathrm{WI}}$, day-to-day variation in water disappearance

684 VAR ${ }_{W D}$, day-to-day variation in water dispensed

685 VAR wIDUR, day-to-day variation in water intake duration

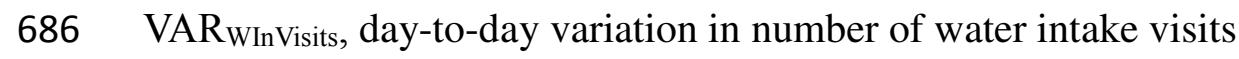

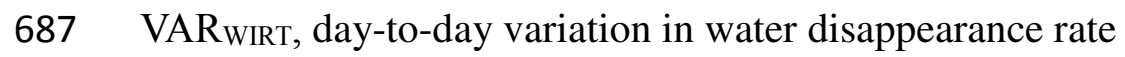

688 VAR ${ }_{W D R T}$, day-to-day variation in water dispense rate

689 WIDUR, average daily water intake duration

690 WInVisits, average number of daily water intake visits

691 WIRT, average daily water disappearance rate

692 WDRT, average daily water dispensed rate

693 WIDUR|FIDUR, ratio trait by fitting FIDUR as covariate for WIDUR

694 WInVisits|FInVisits, ratio trait by fitting FInVisits as covariate for WInVisits

695

696 Declarations

697 Ethics approval and consent to participate

698 Data used herein were obtained in the study described by Cheng et al. (2020), with the

699 appropriate approvals.

700 Consent for publication

$701 \quad$ Not applicable

702 Availability of data and materials

703 Data used are on commercially owned animals and are, therefore, not publicly available. Data

704 are, however, available from the authors upon reasonable request. 


\section{Competing interests}

706 None

707 Funding

708 The data used were from a study that was funded by Genome Canada, Genome Alberta, and

709 PigGen Canada. JC was funded in part by USDA-NIFA grant \# 2017-67007-26144.

\section{Authors' contributions}

711 JC analyzed the data and wrote the manuscript with help from JD. AP helped with data analysis.

$712 \mathrm{GP}, \mathrm{MD}, \mathrm{PC}, \mathrm{JH}, \mathrm{FF}$, and JD designed the project and developed protocols for animal sourcing,

713 management, and phenotype recording in collaboration with members of PigGen Canada. JH

714 was in charge of veterinary oversight on the project. GP was in charge of the database and

715 genotyping for the project. FF was the lead on day-to-day data collection and scheduling. All

716 authors helped with interpretation of the results and reviewed and approved the final manuscript.

\section{Acknowledgements}

718 Members of PigGen Canada are acknowledged for providing the pigs and for helpful discussions, 719 including: Canadian Centre for Swine Improvement, Fast Genetics, Genesus, Hypor, 720 ALPHAGENE, Topigs Norsvin, DNA Genetics, the Canadian Swine Breeders Association, and 721 Alliance Genetics Canada.

\section{LITERATURE CITED}

724 Albers G.A.A., Gray G.D., Piper L.R., Barker J.S.F., Lejambre L.F. \& Barger I.A. The genetics of 725 resistance and resilience to Haemonchus contortus infection in young merino sheep. Int. J. 726 Parasitol. 1987. 17, 1355-1363. 
728 Bisset S.A. and Morris C.A. 1996. Feasibility and implications of breeding sheep for resilience to 729 nematode challenge. Int. J. Parasitol. 1996. 26, 857-868.

730

731 Ahmed, S.T., Mun, H.-S., Yoe, H., Yang, C. J. Monitoring of behavior using a video-recording 732 system for recognition of Salmonella infection in experimentally infected growing pigs. Animal. $7332015 ; 9,115-121$.

734

735 Brew, M.N. Myer R.O. Hersom M.J. Carter J.N. Elzo M.A. Hansen G.R. Riley D.G. Water intake 736 and factors affecting water intake of growing beef cattle. Livest. Sci.2011; 140:297-300.

737

738 Berghof, T. V. L., M. Poppe, and H. A. Mulder. Opportunities to Improve Resilience in Animal 739 Breeding Programs. Front. Genet. 2018. 14.

740

741 Casey, D. S., H. S. Stern, and J. C. M. Dekkers. Identification of errors and factors associated with 742 errors in data from electronic swine feeders. J. Anim. Sci. 2005; 83, 969-982. 743 doi: $10.2527 / 2005.835969 x$.

744

745 Cheng, J., A. M. Putz, J. C. S. Harding, M. K. Dyck, F. Fortin, G. S. Plastow, PigGen Canada, and 746 J. C. M. Dekkers. Genetic analysis of disease resilience in wean-to-finish pigs from a natural 747 disease challenge model. J. Anim. Sci. 2020; skaa244, https://doi.org/10.1093/jas/skaa244. 
749 Dybkjaer, L., Jacobsen A.P., Togersen F.A., Poulsen H.D. Eating and drinking activity of newly 750 weaned piglets: effects of individual characteristics, social mixing, and addition of extra zinc to 751 the feed. J. Anim. Sci. 2006; 84(3):702-11.

752

753 Dufrasne, M., Misztal, I., Tsuruta, S., Gengler, N., and Gray, K. A. Genetic analysis of pig survival 754 up to commercial weight in a crossbred population. Livest. Sci. 2014; 167, 19-24. doi: 755 10.1016/j.livsci.2014.05.001.

756

757 Ding R, Yang M, Wang X, Quan J, Zhuang Z, Zhou S, Li S, Xu Z, Zheng E, Cai G, Liu D., Huang

758 W, Yang J. and Wu Z. Genetic architecture of feeding behavior and feed efficiency in a duroc pig 759 population. Front. Genet. 2018; 9:220. doi: 10.3389/fgene.2018.00220.

760

761 Fortin, F., Turgeon J., Gagnon P., Caron-Simard V. Results and perspectives about automated 762 water intake recording, infrared thermography and visual systems. 2018; BANFF Pork Seminar, 763 Banff, Alberta, Canada, 10-11 January.

764

765 Gilmour. A. R., Gogel, B.J., Cullis, B.R., Welham, S.J., Thompson, R. ASReml user guide release 7664.1 structural specification. 2015; Hemel Hempstead: VSN International. 767

768 Guy, S. Z. Y., Li, L., Thomson, P. C., and Hermesch, S. "Genetic parameters for health of the 769 growing pig using medication records," in Proceeding 11th World Congress of Genetics Applied 770 to Livestock Production, (Auckland). 2018. 
772 Hall, A. D., Hill, W. G., Bampton, P. R., and Webb, A. J. Genetic and phenotypic parameter 773 estimates for feeding pattern and performance tests traits in pigs. Europe PubMed Central, 1999; $774 \quad 68,43-48$.

775

776 Huynh, T.T.T., Aarnonk A.J.A., Verstegen M.W.A., Gerrits W.J.J., Heetkamp M.J.W., Kemp B.,

777 Canh T.T. Effects of increasing temperatures on physiological changes in pigs at different relative 778 humidities. J. Anim. Sci. 2005; 83, 1385-1396.

779

780 Holtkamp, D.J., Kliebenstein, J.B., Neumann, E.J., Zimmerman, J.J., Rotto, H.F., Yoder, T.K.,

781 Wang, C., Yeske, P.E., Mowrer, C.L., Haley, C.A. Assessment of the economic impact of porcine 782 reproductive and respiratory syndrome virus on United States pork producers. J. Swine Health 783 Prod. 2013; 21:72-84.

784

785 IISD. International Institute for Sustainable Development. 2011; http://sdg.iisd.org/news/fao786 world-livestock-report-projects-drastic-increase-in-meat-consumption/ .

787

788 Ivoš, J., Krsnik B. and Kovac`ević S. Ecology and production in pig-breeding. Stoc`arstvo. 1981; $78935,379-416$.

790

791 Kruse, S., Traulsen I., Salau J., Krieter J. A note on using wavelet analysis for disease detection in 792 lactating sows. Comput. Electron. Agric. 2011; 77(1):105-9. 
794 Kashiha, M., Bahr, C., Haredasht, S. A., Ott, S., Moons, C., Niewold, T. A., Odberg, F., et al. The 795 automatic monitoring of pigs water use by cameras. Comput. Electron. Agric. 2013; 90, 164-169.

797 Labroue, F., Gueblez, R., and Sellier, P. Genetic parameters of feeding behaviour and performance 798 traits in group-housed Large White and French Landrace growing pigs. Genet. Sel. Evol. 1997; $79929(4), 451-468$.

800

801 Morgan, C. A., Emmans G.C., Tolkamp B.J., Kyriazakis I. Analysis of the feeding behavior of 802 pigs using different models. Physiol. Behav. 2000; 68, 395-403.

804 Misztal, I., Tsuruta, S., Strabel, T., Auvray, B., Druet, T., and Lee, D. H. "BLUPF90 and related 805 programs (BGF90)," in Procceding 7th World Congress of Genetics Applied to Livestock 806 Production, (Montpellier). 2002.

807

808 McSweeny, J. M., Hermesch, S., and Crump, R. Genetic analysis of feeding pattern traits in pigs. 809 Animal Genetics and Breeding Unit, a Joint Venture of the University of New England and NSW 810 Agriculture, University of New England, Armidale, NSW, 2003; 2351.

811

812 Maselyne, J., I. Adriaens, T. Huybrechts, B. De Ketelaere, S. Millet, J. Vangeyte, A. Van Nuffel, 813 W. Saeys. Measuring the drinking behaviour of individual pigs housed in group using radio 814 frequency identification (RFID). Animal. 2015; pp. 1-10. 
816 Pedersen, B. K., Madsen T.N. Monitoring water intake in pigs: prediction of disease and stressors.

817 In Stowell RR, Bucklin R, Bottcher RW, editors. Proceedings of the Sixth International Livestock

818 Environment Symposium. Amer. Soc. Ag. Eng., St. Joseph, MI, p. 173-9. 2001.

819

820 Paarlberg. Updated estimated economic welfare impacts of Porcine Epidemic Diarrhea virus

821 (PEDV) Department of Agricultural Economics, Purdue University, 2014; Working Paper \#14-4

822

823 Putz, A. M., Harding J.C.S., Dyck M.K., Fortin F., Plastow G.S., Dekkers J.C.M. and PigGen

824 Canada. Novel resilience phenotypes using feed intake data from a natural disease challenge model 825 in wean-to-finish pigs. Front. Genet. 2019; 9:660. doi: 10.3389/fgene.2018.00660.

826

827 Stockill, P. Water: The neglected nutrient. Large Animal Veterinarian July/August. 1991.

828

829 Schulze, V., Roehe, R., Bermejo, J. L., Looft, H., \& Kalm, E. The influence of feeding behaviour 830 on feed intake curve parameters and performance traits of stationtested boars. Livest. Prod. Sci., $831 \quad 2003 ; 82,105-116$.

832

833 UNEP. The critical role of global food consumption patterns in achieving sustainable food systems 834 and food for all. 2012; http://www.humanmedia.org/dcc/pdf/unep_food_report_2012.pdf

835

836 VanRaden, P. Efficient methods to compute genomic prediction. J. Dairy Sci.2008; 91, 4414-4423.

837 doi: 10.3168/jds.2007-0980 
839 Young, J. M., Dekkers, J., Gabler, N., Johnson, A., Nettleton, D., Rothschild, M. , and Tuggle, C.

840 The effect of selection for residual feed intake during the grow/finish phase of production on

841 feeding behavior traits and sow reproduction and lactation efficiency in yorkshire pigs. $\mathrm{PhD}$

842 Dissertation. 2012; Iowa State University, Ames.

843 
844

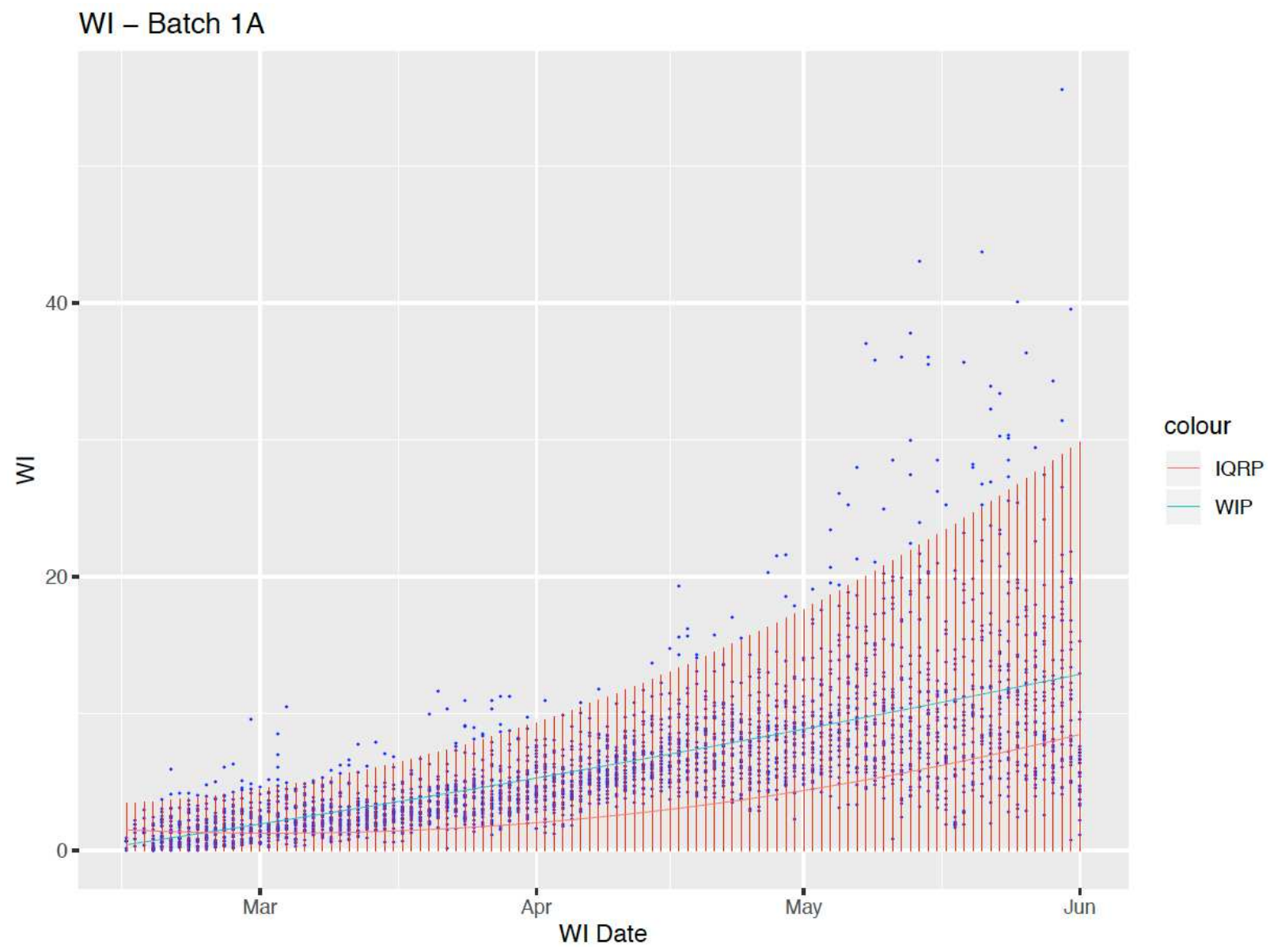

Figure 1. Outlier detection for water disappearance (WI) for a random batch of pigs based on

848 predicted mean (WIP) + 2 times the predicted interquartile range (IQRP).

849

850

851

852 


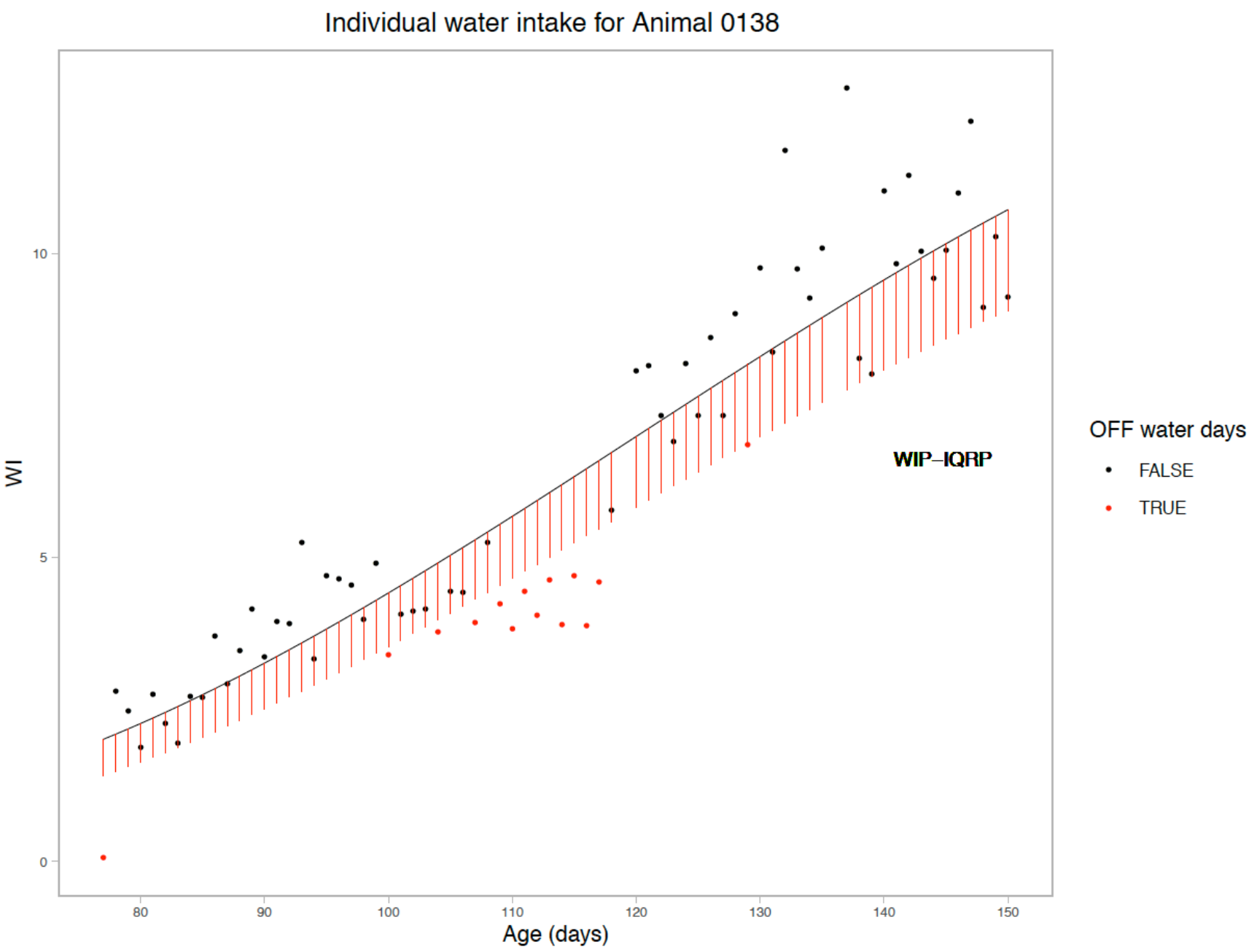
based on a quadratic regression model; daily water intake data that had residuals less than the

861 predicted interquartile range were considered off-water days. 
866

867 Table 1. Summary statistics and estimates (SE in parentheses) of heritability $\left(\mathrm{h}^{2}\right)$ and litter effects $868\left(\mathrm{c}^{2}\right)$ for water and feed intake and behavior traits

\begin{tabular}{|c|c|c|c|c|c|c|}
\hline Trait & No. records & Mean & SD & $\mathrm{CV}$ & $\mathrm{h}^{2}(\mathrm{SE})$ & $\mathrm{c}^{2}(\mathrm{SE})$ \\
\hline \multicolumn{7}{|l|}{ Water intake } \\
\hline $\operatorname{ADWI}(\mathrm{L} / \mathrm{d})$ & 1,379 & 5.06 & 2.42 & 0.48 & $0.34(0.08)$ & $0.05(0.04)$ \\
\hline ADWD (L/d) & 1,426 & 4.84 & 3.30 & 0.68 & $0.44(0.07)$ & $0.04(0.04)$ \\
\hline WIDUR (min/d) & 1,525 & 14.73 & 4.37 & 0.30 & $0.54(0.07)$ & $0.06(0.03)$ \\
\hline WInVisits & 1,598 & 31.32 & 7.49 & 0.24 & $0.58(0.06)$ & $0.01(0.03)$ \\
\hline WIRT (L/min) & 1,529 & 0.36 & 0.13 & 0.36 & $0.25(0.06)$ & $0.00(0.00)$ \\
\hline WDRT (L/min) & 1,533 & 0.32 & 0.18 & 0.56 & $0.30(0.07)$ & $0.02(0.03)$ \\
\hline ADWI|ADFI & 1,379 & - & - & - & $0.42(0.08)$ & $0.02(0.04)$ \\
\hline ADWD $\mid$ ADFI & 1,426 & - & - & - & $0.50(0.07)$ & $0.02(0.03)$ \\
\hline WIDUR|FIDUR & 1,525 & - & - & - & $0.56(0.07)$ & $0.05(0.03)$ \\
\hline WInVisits|FInVisits & 1,598 & - & - & - & $0.60(0.06)$ & $0.02(0.03)$ \\
\hline \multicolumn{7}{|l|}{ Feed intake } \\
\hline $\operatorname{ADFI}(\mathrm{kg} / \mathrm{d})$ & 2,337 & 2.20 & 0.32 & 0.15 & $0.35(0.05)$ & $0.03(0.03)$ \\
\hline FIDUR $(\mathrm{min} / \mathrm{d})$ & 2,337 & 59.51 & 11.49 & 0.19 & $0.49(0.06)$ & $0.04(0.03)$ \\
\hline FInVisits & 2,337 & 17.71 & 6.79 & 0.38 & $0.51(0.05)$ & $0.08(0.03)$ \\
\hline FIRT $(\mathrm{kg} / \mathrm{min})$ & 2,337 & 0.038 & 0.009 & 0.25 & $0.45(0.05)$ & $0.03(0.03)$ \\
\hline ADFI|ADWI & 2,337 & - & - & - & $0.42(0.06)$ & $0.00(0.00)$ \\
\hline ADFI|ADWD & 2,337 & - & - & - & $0.42(0.06)$ & $0.00(0.00)$ \\
\hline FIDUR|WIDUR & 2,337 & - & - & - & $0.55(0.07)$ & $0.02(0.03)$ \\
\hline FInVisits|WInVisits & 2,337 & - & - & - & $0.46(0.07)$ & $0.12(0.04)$ \\
\hline \multicolumn{7}{|l|}{ Water intake } \\
\hline $\mathrm{VAR}_{W I}$ & 1,379 & 2.08 & 1.41 & $0.29^{1}$ & $0.35(0.07)$ & $0.00(0.00)$ \\
\hline $\mathrm{VAR}_{W D}$ & 1,426 & 2.51 & 1.66 & $0.33^{1}$ & $0.38(0.08)$ & $0.01(0.04)$ \\
\hline VAR $_{\text {WIDUR }}$ & 1,525 & 4.84 & 2.06 & $0.15^{1}$ & $0.25(0.07)$ & $0.13(0.04)$ \\
\hline VARWInVisits & 1,598 & 8.83 & 2.74 & $0.09^{1}$ & $0.33(0.07)$ & $0.06(0.04)$ \\
\hline VAR WIRT & 1,529 & 0.11 & 0.04 & $0.92^{1}$ & $0.22(0.06)$ & $0.00(0.00)$ \\
\hline VAR $_{W D R T}$ & 1,533 & 0.12 & 0.05 & $1.08^{1}$ & $0.22(0.07)$ & $0.00(0.00)$ \\
\hline \multicolumn{7}{|l|}{ Feed intake } \\
\hline $\mathrm{VAR}_{\mathrm{FI}}$ & 2,337 & 0.50 & 0.10 & $0.67^{1}$ & $0.08(0.04)$ & $0.02(0.03)$ \\
\hline VAR $_{\text {FIDUR }}$ & 2,337 & 13.35 & 3.79 & $0.06^{1}$ & $0.23(0.05)$ & $0.02(0.03)$ \\
\hline VAR $_{\text {FInVisits }}$ & 2,292 & 6.12 & 2.46 & $0.14^{1}$ & $0.47(0.06)$ & $0.08(0.03)$ \\
\hline VAR $_{\text {FIRT }}$ & 2,292 & 0.058 & 0.006 & $2.50^{1}$ & $0.00(0.00)$ & $0.04(0.03)$ \\
\hline \multicolumn{7}{|l|}{ Water intake } \\
\hline $\mathrm{OFF}_{\mathrm{WI}}$ & 1,308 & 0.12 & 0.08 & 0.67 & $0.27(0.07)$ & $0.05(0.04)$ \\
\hline $\mathrm{OFF}_{\mathrm{WD}}$ & 1,315 & 0.11 & 0.09 & 0.81 & $0.21(0.07)$ & $0.01(0.04)$ \\
\hline OFF $_{\text {WIDUR }}$ & 1,517 & 0.11 & 0.06 & 0.55 & $0.15(0.06)$ & $0.10(0.04)$ \\
\hline OFF $_{\text {WInVisits }}$ & 1,595 & 0.12 & 0.06 & 0.50 & $0.31(0.07)$ & $0.01(0.03)$ \\
\hline OFF $_{\text {WIRT }}$ & 1,515 & 0.11 & 0.07 & 0.64 & $0.17(0.06)$ & $0.00(0.00)$ \\
\hline OFF $_{\text {WDRT }}$ & 1,483 & 0.11 & 0.08 & 0.73 & $0.16(0.06)$ & $0.00(0.00)$ \\
\hline \multicolumn{7}{|l|}{ Feed intake } \\
\hline $\mathrm{OFF}_{\mathrm{FI}}$ & 2,337 & 0.04 & 0.05 & 1.25 & $0.10(0.04)$ & $0.05(0.03)$ \\
\hline
\end{tabular}




\begin{tabular}{rrrrrrr}
\hline OFFFIDUR & 2,337 & 0.04 & 0.04 & 1.00 & $0.26(0.05)$ & $0.01(0.02)$ \\
OFF FInVisits & 2,292 & 0.05 & 0.07 & 1.40 & $0.13(0.04)$ & $0.04(0.03)$ \\
OFFFIRT & 2,292 & 0.05 & 0.13 & 2.60 & $0.13(0.05)$ & $0.01(0.03)$ \\
\hline
\end{tabular}

869 CV: coefficient of variation; ADWI: average daily water disappearance; ADWD: average daily

870 water dispensed; WIDUR: average daily water intake duration; WInVisits: average number of

871 daily water intake visits; WIRT: water disappearance rate; WDRT: water dispensed rate; ADFI:

872 average daily feed intake; FIDUR: average daily feed intake duration; FInVisits: average daily

873 feed intake visits; FIRT: feed intake rate; VAR ${ }_{W I}, V_{\text {VAR }}$, VAR ${ }_{W I D U R}, V_{\text {VAR }}$ Wisits, $_{\text {VAR }}$ WIRT,

874 VAR $_{\text {WDRT, }}, \mathrm{VAR}_{\mathrm{FI}}, \mathrm{VAR}_{\mathrm{FIDUR}}, \mathrm{VAR}_{\mathrm{FInV}}$ isits, and $\mathrm{VAR}_{\mathrm{FIRT}}$ are the day-to-day variation for

875 corresponding drinking and feeding traits; $\mathrm{OFF}_{\mathrm{WI}}, \mathrm{OFF}_{\mathrm{WD}}, \mathrm{OFF}_{\mathrm{WIDUR}}, \mathrm{OFF}_{\mathrm{WInVisits}}, \mathrm{OFF}_{\mathrm{WIRT}}$,

$876 \mathrm{OFF}_{\mathrm{WDRT}}, \mathrm{OFF}_{\mathrm{FI}}, \mathrm{OFF}_{\mathrm{FIDUR}}, \mathrm{OFF}_{\mathrm{FInV}} \mathrm{isits}$, and $\mathrm{OFF}_{\mathrm{FIRT}}$ are the proportion of off days for

877 corresponding drinking and feeding traits

$878{ }^{1}$ Coefficients of day-to-day variation, calculated as the SD of VAR of the trait divided by the mean

879 of the corresponding trait, e.g. $\sqrt{\mathrm{VAR}_{F I}} / \mathrm{ADFI}$;

880

881

882

883

884

885

886

887

888

889

890 
892 Table 2. Percentages of observations with off-feed, off-water, and treatment events (on diagonal) 893 and of observed (upper diagonal) and expected (with independence, below diagonal) concurrent 894 off-feed and off-water days and days pigs received treatment on a daily and a 7-day window basis.

\begin{tabular}{|c|c|c|c|}
\hline On a daily basis & Off-feed & Off-water & Treatment \\
\hline Off-feed & 2.48 & 0.96 & 0.24 \\
\hline Off-water & 0.24 & 12.50 & 0.29 \\
\hline Treatment & 0.02 & 0.12 & 0.88 \\
\hline
\end{tabular}

On a 7-day window basis

$\begin{array}{cccc}\text { Off-feed } & \mathbf{9 . 7 2} & 5.82 & 2.67 \\ \text { Off-water } & 4.09 & \mathbf{4 1 . 1 0} & 3.71 \\ \text { Treatment } & 0.60 & 2.45 & \mathbf{5 . 9 3}\end{array}$

895 All observed percentages of concurrent events (above diagonal) were greater than expected (below 896 diagonal) based on a Chi-square test at $\mathrm{p}<0.0001$. 
Table 3. Estimates of genetic (upper triangle) and phenotypic (lower triangle) correlations (SE in parentheses) among drinking behavior and among feeding behavior traits

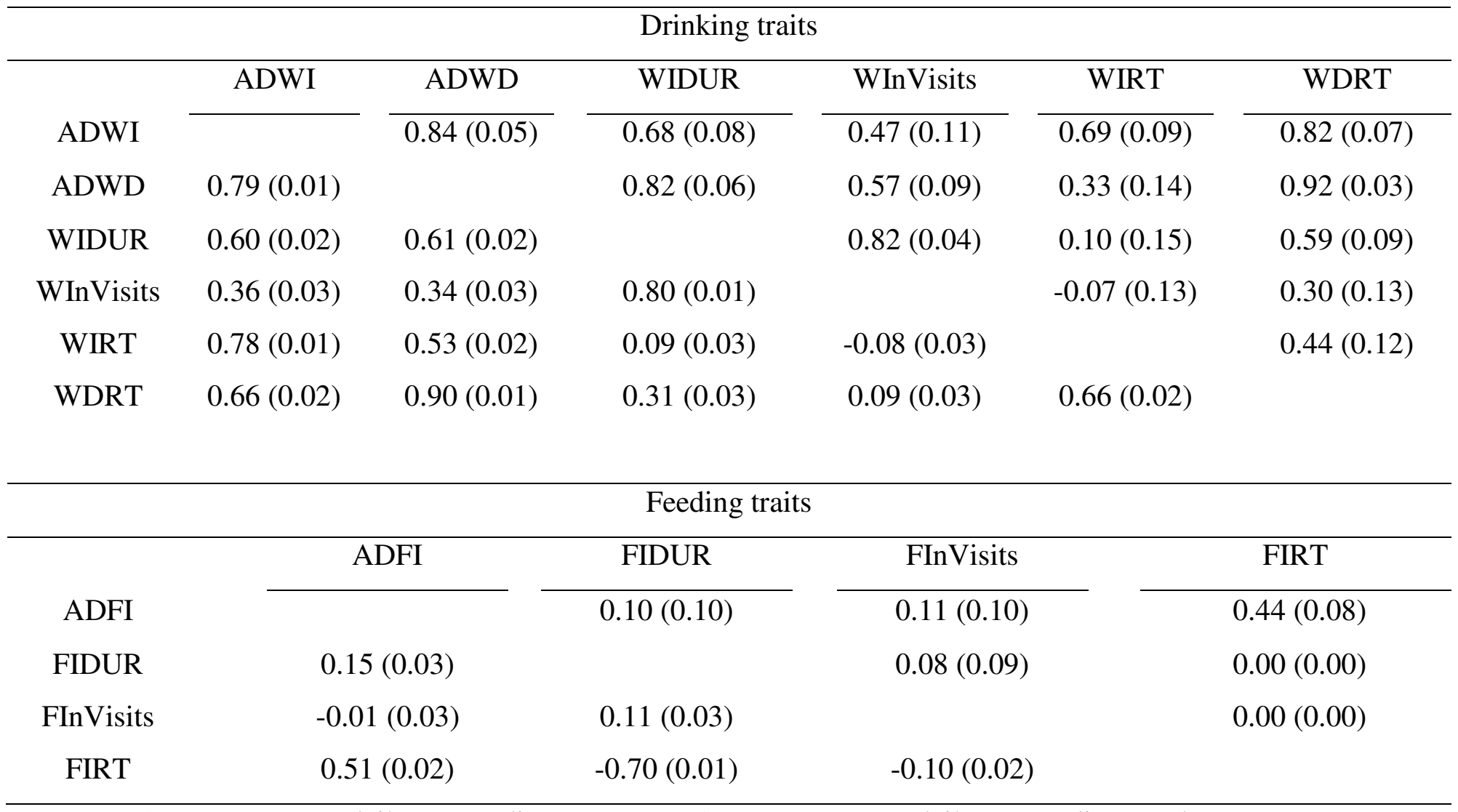

ADWI: average daily water disappearance; ADWD: average daily water dispensed; WIDUR:

average daily water intake duration; WInVisits: average number of daily water intake visits; WIRT:

water disappearance rate; WDRT: water dispensed rate; ADFI: average daily feed intake; FIDUR:

average daily feed intake duration; FInVisits: average number of daily feed intake visits; FIRT:

feed intake rate. 
Table 4. Estimates of phenotypic and genetic correlations (SE in parentheses) between corresponding drinking and feeding behavior traits

\begin{tabular}{|c|c|c|c|}
\hline Drinking trait & Feeding trait & Genetic & Phenotypic \\
\hline ADWI & ADFI & $0.36(0.10)$ & $0.39(0.03)$ \\
\hline ADWD & ADFI & $0.23(0.10)$ & $0.29(0.03)$ \\
\hline WIDUR & FIDUR & $0.10(0.09)$ & $0.16(0.03)$ \\
\hline WInVisits & FInVisits & $0.24(0.08)$ & $0.29(0.03)$ \\
\hline WIRT & FIRT & $0.24(0.12)$ & $0.27(0.03)$ \\
\hline WDRT & FIRT & $0.08(0.11)$ & $0.13(0.03)$ \\
\hline VAR $_{W I}$ & VAR $_{\mathrm{FI}}$ & $0.10(0.23)$ & $0.05(0.03)$ \\
\hline $\mathrm{VAR}_{W D}$ & VAR $_{F I}$ & $-0.08(0.23)$ & $0.03(0.03)$ \\
\hline VAR WIDUR & VAR $_{\text {FIDUR }}$ & $-0.14(0.14)$ & $0.08(0.03)$ \\
\hline VAR WInVisits $_{\text {}}$ & VAR $_{\text {FInVisits }}$ & $0.18(0.10)$ & $0.13(0.03)$ \\
\hline VAR ${ }_{W I R T}$ & VAR $_{\text {FIRT }}$ & $-0.06(1.58)$ & $0.16(0.05)$ \\
\hline VAR WDRT & VAR $_{\text {FIRT }}$ & $0.64(2.08)$ & $-0.02(0.03)$ \\
\hline $\mathrm{OFF}_{\mathrm{WI}}$ & $\mathrm{OFF}_{\mathrm{FI}}$ & $-0.25(0.20)$ & $-0.10(0.03)$ \\
\hline $\mathrm{OFF}_{W D}$ & $\mathrm{OFF}_{\mathrm{FI}}$ & $0.04(0.24)$ & $-0.14(0.03)$ \\
\hline OFF $_{\text {WIDUR }}$ & $\mathrm{OFF}_{\text {FIDUR }}$ & $-0.29(0.16)$ & $-0.05(0.03)$ \\
\hline $\mathrm{OFF}_{\text {WInVisits }}$ & OFF FInVisits $_{\text {f }}$ & $0.08(0.16)$ & $-0.01(0.03)$ \\
\hline $\mathrm{OFF}_{\mathrm{WIRT}}$ & OFFFIRT $_{\text {FI }}$ & $-0.23(0.25)$ & $-0.12(0.03)$ \\
\hline OFF $_{W D R T}$ & OFF FIRT $_{\text {FI }}$ & $-0.07(0.28)$ & $-0.09(0.03)$ \\
\hline
\end{tabular}

ADWI: average daily water disappearance; ADWD: average daily water dispensed; WIDUR:

average daily water intake duration; WInVisits: average number of daily water intake visits; WIRT: water disappearance rate; WDRT: water dispensed rate; ADFI: average daily feed intake; FIDUR: average daily feed intake duration; FInVisits: average number of daily feed intake visits; FIRT:

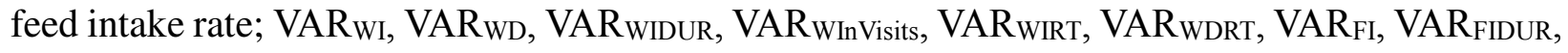
$\mathrm{VAR}_{\mathrm{FInVisits}}$, and $\mathrm{VAR}_{\mathrm{FIRT}}$ are the day-to-day variation for corresponding drinking and feeding traits; $\mathrm{OFF}_{\mathrm{WI}}, \mathrm{OFF}_{\mathrm{WD}}, \mathrm{OFF}_{\mathrm{WIDUR}}, \mathrm{OFF}_{\mathrm{WInV} V i s i t s}, \mathrm{OFF}_{\mathrm{WIRT}}, \mathrm{OFF}_{\mathrm{WDRT}}, \mathrm{OFF}_{\mathrm{FI}}, \mathrm{OFF}_{\mathrm{FIDUR}}, \mathrm{OFF}_{\mathrm{FInV} \text { isits, }}$ and $\mathrm{OFF}_{\text {FIRT }}$ are the proportion of off days for corresponding drinking and feeding traits. 
Table 5. Estimates of phenotypic and genetic correlations (SE in parentheses) of drinking and feeding behavior traits with growth rate (ADG) in three phases

\begin{tabular}{|c|c|c|c|c|c|c|}
\hline \multirow{2}{*}{ Trait } & \multicolumn{2}{|c|}{ Quarantine nursery } & \multicolumn{2}{|c|}{ Challenge nursery } & \multicolumn{2}{|c|}{ Finisher } \\
\hline & Genetic & Phenotypic & Genetic & Phenotypic & Genetic & Phenotypic \\
\hline \multicolumn{7}{|l|}{ Water intake } \\
\hline ADWI & $0.10(0.13)$ & $0.06(0.03)$ & $0.39(0.13)$ & $0.27(0.03)$ & $0.29(0.12)$ & $0.34(0.03)$ \\
\hline ADWD & $0.13(0.11)$ & $0.06(0.03)$ & $0.22(0.12)$ & $0.19(0.03)$ & $0.22(0.11)$ & $0.25(0.03)$ \\
\hline WIDUR & $0.15(0.10)$ & $0.02(0.03)$ & $0.17(0.11)$ & $0.10(0.03)$ & $0.23(0.10)$ & $0.27(0.03)$ \\
\hline WInVisits & $0.04(0.10)$ & $-0.03(0.03)$ & $0.07(0.11)$ & $-0.02(0.03)$ & $0.17(0.10)$ & $0.18(0.03)$ \\
\hline WIRT & $0.14(0.14)$ & $0.10(0.03)$ & $0.34(0.14)$ & $0.31(0.03)$ & $0.19(0.14)$ & $0.27(0.03)$ \\
\hline WDRT & $0.13(0.13)$ & $0.08(0.03)$ & $0.15(0.14)$ & $0.22(0.03)$ & $0.13(0.13)$ & $0.21(0.03)$ \\
\hline ADWI|ADFI & $-0.04(0.12)$ & $-0.02(0.03)$ & $0.24(0.14)$ & $0.15(0.03)$ & $-0.02(0.13)$ & $0.01(0.04)$ \\
\hline ADWD|ADFI & $0.04(0.11)$ & $-0.01(0.03)$ & $0.04(0.13)$ & $0.10(0.03)$ & $0.00(0.12)$ & $-0.01(0.04)$ \\
\hline WIDUR|FIDUR & $0.12(0.10)$ & $0.02(0.03)$ & $0.15(0.11)$ & $0.13(0.11)$ & $0.23(0.10)$ & $0.25(0.03)$ \\
\hline WInVisits|FInVisits & $0.06(0.10)$ & $-0.02(0.03)$ & $0.10(0.11)$ & $0.01(0.03)$ & $0.13(0.10)$ & $0.19(0.03)$ \\
\hline \multicolumn{7}{|l|}{ Feed intake } \\
\hline ADFI & $0.27(0.10)$ & $0.23(0.02)$ & $0.49(0.09)$ & $0.42(0.02)$ & $0.84(0.03)$ & $0.86(0.01)$ \\
\hline FIDUR & $0.10(0.09)$ & $0.01(0.03)$ & $-0.09(0.11)$ & $-0.08(0.03)$ & $0.09(0.10)$ & $0.16(0.03)$ \\
\hline FInVisits & $-0.14(0.08)$ & $-0.10(0.03)$ & $-0.03(0.10)$ & $-0.08(0.03)$ & $-0.01(0.09)$ & $-0.03(0.03)$ \\
\hline FIRT & $0.08(0.09)$ & $0.15(0.03)$ & $0.34(0.09)$ & $0.40(0.02)$ & $0.35(0.09)$ & $0.42(0.02)$ \\
\hline ADFI|ADWI & $0.27(0.12)$ & $0.21(0.03)$ & $0.49(0.11)$ & $0.46(0.03)$ & $0.82(0.04)$ & $0.86(0.01)$ \\
\hline ADFI|ADWD & $0.27(0.11)$ & $0.22(0.03)$ & $0.54(0.10)$ & $0.48(0.02)$ & $0.80(0.04)$ & $0.86(0.01)$ \\
\hline FIDUR/WIDUR & $0.12(0.10)$ & $0.01(0.03)$ & $-0.12(0.12)$ & $-0.16(0.03)$ & $-0.01(0.11)$ & $0.11(0.03)$ \\
\hline FInVisits|WInVisits & $-0.22(0.10)$ & $-0.09(0.03)$ & $-0.13(0.12)$ & $-0.10(0.03)$ & $0.03(0.11)$ & $-0.06(0.03)$ \\
\hline \multicolumn{7}{|l|}{ Water intake } \\
\hline $\mathrm{VAR}_{\mathrm{WI}}$ & $0.10(0.13)$ & $-0.01(0.03)$ & $0.23(0.15)$ & $0.15(0.03)$ & $0.21(0.13)$ & $0.17(0.03)$ \\
\hline $\mathrm{VAR}_{\mathrm{WD}}$ & $0.05(0.13)$ & $0.00(0.03)$ & $0.10(0.14)$ & $0.12(0.03)$ & $0.20(0.13)$ & $0.15(0.03)$ \\
\hline VAR $_{\text {WIDUR }}$ & $0.09(0.12)$ & $0.01(0.03)$ & $0.03(0.14)$ & $0.08(0.03)$ & $0.27(0.12)$ & $0.12(0.03)$ \\
\hline VAR $_{\text {WInVisits }}$ & $0.01(0.12)$ & $-0.02(0.03)$ & $-0.07(0.14)$ & $0.03(0.03)$ & $0.08(0.13)$ & $-0.01(0.03)$ \\
\hline VAR $_{\text {WIRT }}$ & $0.10(0.15)$ & $0.04(0.03)$ & $0.13(0.16)$ & $0.14(0.03)$ & $0.09(0.16)$ & $0.14(0.03)$ \\
\hline VAR $_{\text {WDRT }}$ & $0.13(0.15)$ & $0.04(0.03)$ & $0.14(0.17)$ & $0.13(0.03)$ & $0.13(0.15)$ & $0.13(0.03)$ \\
\hline \multicolumn{7}{|l|}{ Feed intake } \\
\hline $\mathrm{VAR}_{\mathrm{FI}}$ & $0.55(0.20)$ & $0.15(0.02)$ & $0.65(0.27)$ & $0.17(0.02)$ & $0.30(0.22)$ & $-0.04(0.02)$ \\
\hline $\mathrm{VAR}_{\mathrm{FIDUR}}$ & $0.11(0.12)$ & $0.03(0.03)$ & $-0.31(0.13)$ & $-0.18(0.02)$ & $-0.23(0.12)$ & $-0.31(0.02)$ \\
\hline VAR $_{\text {FInVisits }}$ & $-0.11(0.09)$ & $-0.06(0.03)$ & $-0.20(0.11)$ & $-0.09(0.02)$ & $0.01(0.10)$ & $-0.12(0.02)$ \\
\hline $\mathrm{VAR}_{\mathrm{FIRT}}$ & $0.40(0.87)$ & $0.00(0.02)$ & $-0.37(1.25)$ & $-0.03(0.02)$ & $0.16(0.86)$ & $-0.08(0.02)$ \\
\hline \multicolumn{7}{|l|}{ Water intake } \\
\hline $\mathrm{OFF}_{\mathrm{WI}}$ & $0.03(0.14)$ & $0.05(0.03)$ & $0.38(0.15)$ & $0.19(0.03)$ & $0.19(0.14)$ & $0.16(0.03)$ \\
\hline $\mathrm{OFF}_{\mathrm{WD}}$ & $0.11(0.15)$ & $0.03(0.03)$ & $0.01(0.18)$ & $0.15(0.03)$ & $-0.05(0.17)$ & $0.16(0.03)$ \\
\hline OFF $_{\text {WIDUR }}$ & $0.18(0.14)$ & $0.03(0.03)$ & $0.12(0.16)$ & $0.06(0.03)$ & $0.11(0.15)$ & $0.04(0.03)$ \\
\hline OFF $_{\text {WInVisits }}$ & $0.10(0.13)$ & $0.00(0.03)$ & $-0.03(0.14)$ & $0.01(0.03)$ & $-0.04(0.13)$ & $-0.06(0.03)$ \\
\hline OFF $_{\text {WIRT }}$ & $-0.02(0.18)$ & $0.04(0.03)$ & $0.31(0.19)$ & $0.19(0.03)$ & $0.00(0.19)$ & $0.10(0.03)$ \\
\hline OFF $_{\text {WDRT }}$ & $0.24(0.18)$ & $0.07(0.03)$ & $0.24(0.21)$ & $0.16(0.03)$ & $0.18(0.18)$ & $0.14(0.03)$ \\
\hline \multicolumn{7}{|l|}{ Feed intake } \\
\hline $\mathrm{OFF}_{\mathrm{FI}}$ & $-0.15(0.17)$ & $-0.07(0.02)$ & $-0.35(0.17)$ & $-0.21(0.02)$ & $-0.80(0.08)$ & $-0.71(0.01)$ \\
\hline
\end{tabular}




\begin{tabular}{rlccccc} 
OFFFIDUR $_{\text {FIn }}$ & $0.02(0.13)$ & $0.03(0.03)$ & $0.03(0.14)$ & $-0.02(0.03)$ & $-0.51(0.10)$ & $-0.46(0.02)$ \\
OFFF FInVisits & $0.36(0.13)$ & $0.12(0.02)$ & $0.08(0.16)$ & $0.10(0.03)$ & $-0.01(0.15)$ & $-0.07(0.02)$ \\
OFFFFT $_{\text {FIRT }}$ & $0.03(0.15)$ & $-0.03(0.02)$ & $-0.39(0.16)$ & $-0.24(0.02)$ & $-0.42(0.13)$ & $-0.47(0.02)$ \\
\hline
\end{tabular}

ADWI: average daily water disappearance; ADWD: average daily water dispensed; WIDUR:

average daily water intake duration; WInVisits: average number of daily water intake visits; WIRT:

water disappearance rate; WDRT: water dispensed rate; ADFI: average daily feed intake; FIDUR:

average daily feed intake duration; FInVisits: average number of daily feed intake visits; FIRT:

feed intake rate; ADWI|ADFI, ADWD|ADFI, WIDUR/FIDUR, WInVisits|FInVisits, ADFI|ADWI, ADFI|ADWD, FIDUR|WIDUR, FInVisits|WInVisits are the fitting the second trait as covariate for the first trait; VAR ${ }_{W I}, V_{W D}, V_{W I D U R}, V_{W} R_{W I n V i s i t s}, V_{W} R_{W I R T}, V_{W A R T}$,

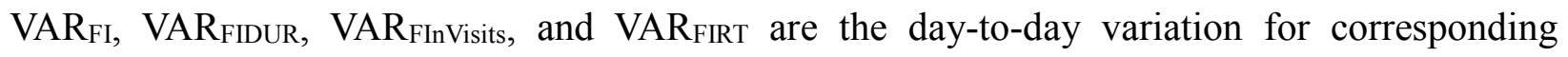
drinking and feeding traits; OFF ${ }_{\mathrm{WI}}, \mathrm{OFF}_{\mathrm{WD}}, \mathrm{OFF}_{\mathrm{WIDUR}}, \mathrm{OFF}_{\mathrm{WInVisits}}, \mathrm{OFF}_{\mathrm{WIRT}}, \mathrm{OFF}_{\mathrm{WDRT}}, \mathrm{OFF}_{\mathrm{FI}}$, $\mathrm{OFF}_{\mathrm{FIDUR}}, \mathrm{OFF}_{\mathrm{FInVisits}}$, and OFFFIRT are the proportion of off days for corresponding drinking and feeding traits. 
Table 6. Estimates of phenotypic correlations (SE in parentheses) of drinking and feeding behaviors with health scores, treatment rates, and mortality in different phases

\begin{tabular}{|c|c|c|c|c|c|}
\hline \multirow{2}{*}{ Trait } & \multicolumn{2}{|c|}{ Health score } & \multicolumn{3}{|c|}{ Treatment rate } \\
\hline & cNursery & Finisher & cNursery & Finisher & Combined \\
\hline \multicolumn{6}{|l|}{ Water intake } \\
\hline ADWI & $0.11(0.03)$ & $0.19(0.03)$ & $-0.13(0.03)$ & $-0.03(0.03)$ & $-0.12(0.03)$ \\
\hline ADWD & $0.03(0.03)$ & $0.13(0.03)$ & $-0.11(0.03)$ & $-0.02(0.03)$ & $-0.09(0.03)$ \\
\hline WIDUR & $0.00(0.03)$ & $0.03(0.03)$ & $-0.11(0.03)$ & $-0.02(0.03)$ & $-0.09(0.03)$ \\
\hline WInVisits & $-0.04(0.03)$ & $-0.04(0.03)$ & $-0.10(0.03)$ & $-0.04(0.03)$ & $-0.10(0.03)$ \\
\hline WIRT & $0.16(0.03)$ & $0.26(0.03)$ & $-0.13(0.03)$ & $-0.07(0.03)$ & $-0.13(0.03)$ \\
\hline WDRT & $0.06(0.03)$ & $0.18(0.03)$ & $-0.12(0.03)$ & $-0.04(0.03)$ & $-0.11(0.03)$ \\
\hline ADWI|ADFI & $0.05(0.03)$ & $0.03(0.03)$ & $-0.06(0.03)$ & $0.04(0.03)$ & $-0.01(0.03)$ \\
\hline ADWD $\mid$ ADFI & $-0.01(0.03)$ & $0.02(0.03)$ & $-0.06(0.03)$ & $0.03(0.03)$ & $-0.02(0.03)$ \\
\hline WIDUR|FIDUR & $0.01(0.03)$ & $0.03(0.03)$ & $-0.12(0.03)$ & $-0.02(0.03)$ & $-0.09(0.03)$ \\
\hline WInVisits|FInVisits & $-0.05(0.03)$ & $-0.03(0.03)$ & $-0.10(0.03)$ & $-0.04(0.03)$ & $-0.09(0.03)$ \\
\hline \multicolumn{6}{|l|}{ Feed intake } \\
\hline ADFI & $0.14(0.02)$ & $0.39(0.02)$ & $-0.27(0.02)$ & $-0.18(0.02)$ & $-0.30(0.02)$ \\
\hline FIDUR & $-0.03(0.02)$ & $0.02(0.02)$ & $0.00(0.02)$ & $-0.03(0.02)$ & $-0.01(0.02)$ \\
\hline FInVisits & $-0.03(0.02)$ & $-0.05(0.02)$ & $-0.02(0.02)$ & $-0.01(0.02)$ & $-0.03(0.02)$ \\
\hline FIRT & $0.12(0.02)$ & $0.24(0.02)$ & $-0.17(0.02)$ & $-0.09(0.02)$ & $-0.17(0.02)$ \\
\hline ADFI|ADWI & $0.15(0.03)$ & $0.37(0.03)$ & $-0.22(0.03)$ & $-0.17(0.13)$ & $-0.25(0.03)$ \\
\hline ADFI|ADWD & $0.17(0.03)$ & $0.38(0.03)$ & $-0.24(0.03)$ & $-0.16(0.03)$ & $-0.25(0.03)$ \\
\hline FIDUR/WIDUR & $-0.06(0.03)$ & $0.00(0.03)$ & $0.03(0.03)$ & $-0.01(0.03)$ & $0.01(0.03)$ \\
\hline FInVisits|WInVisits & $0.01(0.03)$ & $-0.03(0.03)$ & $0.01(0.03)$ & $-0.01(0.03)$ & $-0.01(0.03)$ \\
\hline \multicolumn{6}{|l|}{ Water intake } \\
\hline VAR $_{W I}$ & $0.04(0.03)$ & $0.12(0.03)$ & $-0.06(0.03)$ & $0.00(0.03)$ & $-0.04(0.03)$ \\
\hline VAR $_{W D}$ & $0.01(0.03)$ & $0.09(0.03)$ & $-0.07(0.03)$ & $0.02(0.03)$ & $-0.04(0.03)$ \\
\hline VAR $_{\text {WIDUR }}$ & $0.03(0.03)$ & $0.04(0.03)$ & $-0.06(0.03)$ & $0.03(0.03)$ & $-0.02(0.03)$ \\
\hline VAR $_{\text {WInVisits }}$ & $-0.01(0.03)$ & $-0.04(0.03)$ & $0.03(0.03)$ & $0.06(0.03)$ & $0.05(0.03)$ \\
\hline VAR & $0.06(0.03)$ & $0.19(0.03)$ & $-0.08(0.03)$ & $-0.04(0.03)$ & $-0.09(0.03)$ \\
\hline VAR $_{W D R T}$ & $0.04(0.03)$ & $0.15(0.03)$ & $-0.07(0.03)$ & $-0.04(0.03)$ & $-0.08(0.03)$ \\
\hline \multicolumn{6}{|l|}{ Feed intake } \\
\hline VAR $_{\text {FI }}$ & $0.10(0.02)$ & $0.09(0.02)$ & $0.18(0.02)$ & $0.03(0.02)$ & $0.15(0.02)$ \\
\hline VAR $_{\text {FIDUR }}$ & $-0.03(0.02)$ & $-0.21(0.02)$ & $0.23(0.02)$ & $0.19(0.02)$ & $0.27(0.02)$ \\
\hline VAR $_{\text {FInVisits }}$ & $-0.03(0.03)$ & $-0.11(0.02)$ & $0.06(0.02)$ & $0.09(0.02)$ & $0.07(0.02)$ \\
\hline VAR $_{\text {FIRT }}$ & $-0.01(0.02)$ & $-0.06(0.02)$ & $0.01(0.02)$ & $0.05(0.02)$ & $0.04(0.02)$ \\
\hline \multicolumn{6}{|l|}{ Water intake } \\
\hline $\mathrm{OFF}_{\mathrm{WI}}$ & $0.05(0.03)$ & $0.14(0.03)$ & $0.00(0.03)$ & $0.03(0.03)$ & $0.01(0.03)$ \\
\hline $\mathrm{OFF}_{\mathrm{WD}}$ & $0.05(0.03)$ & $0.10(0.03)$ & $-0.07(0.03)$ & $-0.04(0.03)$ & $-0.06(0.03)$ \\
\hline OFF $_{\text {WIDUR }}$ & $0.00(0.03)$ & $-0.03(0.03)$ & $-0.04(0.03)$ & $0.06(0.03)$ & $0.01(0.03)$ \\
\hline $\mathrm{OFF}_{\text {WInVisits }}$ & $0.00(0.03)$ & $-0.06(0.03)$ & $0.07(0.03)$ & $0.07(0.03)$ & $0.08(0.03)$ \\
\hline $\mathrm{OFF}_{\text {WIRT }}$ & $0.08(0.03)$ & $0.20(0.03)$ & $-0.05(0.03)$ & $-0.01(0.03)$ & $-0.05(0.03)$ \\
\hline
\end{tabular}




\begin{tabular}{|c|c|c|c|c|c|}
\hline $\mathrm{OFF}_{\text {WDRT }}$ & $0.06(0.03)$ & $0.15(0.03)$ & $-0.08(0.03)$ & $-0.03(0.03)$ & $-0.08(0.03)$ \\
\hline \multicolumn{6}{|l|}{ Feed intake } \\
\hline $\mathrm{OFF}_{\mathrm{FI}}$ & $-0.07(0.02)$ & $-0.35(0.02)$ & $0.31(0.02)$ & $0.25(0.02)$ & $0.38(0.02)$ \\
\hline $\mathrm{OFF}_{\text {FIDUR }}$ & $0.03(0.02)$ & $-0.16(0.02)$ & $0.21(0.02)$ & $0.17(0.02)$ & $0.24(0.02)$ \\
\hline $\mathrm{OFF}_{\text {FInVisits }}$ & $0.03(0.02)$ & $-0.03(0.02)$ & $0.10(0.02)$ & $0.10(0.02)$ & $0.12(0.02)$ \\
\hline OFFFIRT $_{\text {FI }}$ & $-0.10(0.02)$ & $-0.36(0.02)$ & $0.24(0.02)$ & $0.16(0.02)$ & $0.24(0.02)$ \\
\hline
\end{tabular}

cNursery: challenge nursery; Combined: challenge nursery and finisher combined; ADWI: average daily water disappearance; ADWD: average daily water dispensed; WIDUR: average daily water intake duration; WInVisits: average number of daily water intake visits; WIRT: water disappearance rate; WDRT: water dispensed rate; ADFI: average daily feed intake; FIDUR: average daily feed intake duration; FInVisits: average number of daily feed intake visits; FIRT: feed intake rate; ADWI|ADFI, ADWD|ADFI, WIDUR/FIDUR, WInVisits|FInVisits, ADFI|ADWI, ADFI|ADWD, FIDUR|WIDUR, FInVisits|WInVisits are the fitting the second trait

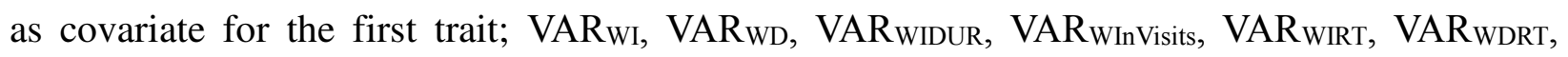
$\mathrm{VAR}_{\mathrm{FI}}, \mathrm{VAR}_{\mathrm{FIDUR}}, \mathrm{VAR}_{\mathrm{FInVisits}}$, and $\mathrm{VAR}_{\mathrm{FIRT}}$ are the day-to-day variation for corresponding drinking and feeding traits; OFF ${ }_{\mathrm{WI}}, \mathrm{OFF}_{\mathrm{WD}}, \mathrm{OFF}_{\mathrm{WIDUR}}, \mathrm{OFF}_{\mathrm{WInVisits}}, \mathrm{OFF}_{\mathrm{WIRT}}, \mathrm{OFF}_{\mathrm{WDRT}}$, OFF $\mathrm{FI}_{\text {, }}$ $\mathrm{OFF}_{\text {FIDUR, }}$ OFF FInVisits, and OFFFIRT are the proportion of off days for corresponding drinking and feeding traits. 
Table 7. Estimates of genetic correlations (SE in parentheses) of drinking and feeding behaviors with health scores, treatment rates, and mortality in different phases

\begin{tabular}{|c|c|c|c|c|c|c|c|}
\hline \multirow{2}{*}{ Trait } & \multicolumn{2}{|c|}{ Health score } & \multicolumn{2}{|c|}{ Treatment rate } & \multicolumn{3}{|c|}{ Mortality } \\
\hline & cNursery & Finisher & cNursery & Combined & cNursery & Finisher & Combined \\
\hline \multicolumn{8}{|l|}{ Water intake } \\
\hline ADWI & $0.09(0.21)$ & $0.30(0.20)$ & $-0.13(0.18)$ & $-0.18(0.24)$ & $-0.18(0.19)$ & $-0.10(0.23)$ & $-0.15(0.17)$ \\
\hline ADWD & $-0.01(0.19)$ & $0.22(0.19)$ & $-0.20(0.16)$ & $-0.24(0.21)$ & $-0.06(0.17)$ & $-0.29(0.20)$ & $-0.20(0.14)$ \\
\hline WIDUR & $-0.21(0.18)$ & $0.10(0.17)$ & $-0.27(0.14)$ & $-0.39(0.19)$ & $-0.10(0.15)$ & $-0.18(0.18)$ & $-0.20(0.13)$ \\
\hline WInVisits & $-0.14(0.17)$ & $-0.18(0.17)$ & $-0.33(0.14)$ & $-0.45(0.19)$ & $-0.24(0.16)$ & $-0.02(0.17)$ & $-0.19(0.13)$ \\
\hline WIRT & $0.41(0.20)$ & $0.36(0.20)$ & $-0.05(0.20)$ & $0.04(0.25)$ & $0.02(0.22)$ & $-0.01(0.23)$ & $0.12(0.18)$ \\
\hline WDRT & $0.15(0.19)$ & $0.24(0.20)$ & $-0.03(0.18)$ & $-0.08(0.23)$ & $0.07(0.20)$ & $-0.20(0.21)$ & $-0.04(0.16)$ \\
\hline ADWI|ADFI & $-0.01(0.14)$ & $0.11(0.21)$ & $-0.19(0.16)$ & $-0.01(0.23)$ & $-0.23(0.18)$ & $-0.10(0.20)$ & $-0.16(0.14)$ \\
\hline ADWD|ADFI & $-0.08(0.13)$ & $0.08(0.19)$ & $-0.16(0.15)$ & $-0.13(0.21)$ & $-0.10(0.16)$ & $-0.25(0.19)$ & $-0.11(0.15)$ \\
\hline WIDUR|FIDUR & $-0.13(0.12)$ & $0.10(0.17)$ & $-0.26(0.13)$ & $-0.37(0.19)$ & $-0.26(0.15)$ & $-0.17(0.18)$ & $-0.20(0.13)$ \\
\hline WInVisits|FInVisits & $-0.13(0.11)$ & $-0.17(0.17)$ & $-0.34(0.13)$ & $-0.48(0.18)$ & $-0.31(0.15)$ & $0.02(0.17)$ & $-0.16(0.12)$ \\
\hline \multicolumn{8}{|l|}{ Feed intake } \\
\hline ADFI & $0.02(0.16)$ & $0.31(0.15)$ & $-0.25(0.14)$ & $-0.33(0.17)$ & $0.02(0.15)$ & $-0.12(0.18)$ & $-0.13(0.20)$ \\
\hline FIDUR & $-0.11(0.16)$ & $0.05(0.16)$ & $-0.03(0.14)$ & $0.00(0.20)$ & $0.40(0.15)$ & $-0.32(0.16)$ & $-0.43(0.18)$ \\
\hline FInVisits & $-0.01(0.15)$ & $-0.13(0.15)$ & $-0.05(0.13)$ & $0.06(0.19)$ & $-0.10(0.14)$ & $-0.27(0.16)$ & $-0.23(0.11)$ \\
\hline FIRT & $0.00(0.14)$ & $0.10(0.15)$ & $-0.06(0.14)$ & $-0.12(0.17)$ & $-0.32(0.15)$ & $-0.02(0.15)$ & $0.10(0.17)$ \\
\hline ADFI|ADWI & $0.20(0.13)$ & $0.36(0.17)$ & $-0.16(0.15)$ & $-0.35(0.19)$ & $0.08(0.18)$ & $0.03(0.20)$ & $-0.03(0.15)$ \\
\hline ADFI|ADWD & $0.23(0.13)$ & $0.37(0.16)$ & $-0.18(0.15)$ & $-0.32(0.19)$ & $0.03(0.17)$ & $-0.02(0.19)$ & $-0.06(0.15)$ \\
\hline FIDUR/WIDUR & $0.10(0.12)$ & $0.01(0.18)$ & $0.02(0.14)$ & $-0.01(0.20)$ & $0.40(0.16)$ & $-0.08(0.18)$ & $0.07(0.13)$ \\
\hline FInVisits|WInVisits & $-0.13(0.13)$ & $-0.01(0.19)$ & $0.20(0.14)$ & $0.28(0.21)$ & $0.00(0.16)$ & $-0.21(0.19)$ & $-0.15(0.14)$ \\
\hline \multicolumn{8}{|l|}{ Water intake } \\
\hline VAR $_{W I}$ & $0.09(0.22)$ & $0.16(0.22)$ & $-0.10(0.19)$ & $-0.15(0.27)$ & $0.00(0.20)$ & $-0.10(0.22)$ & $-0.06(0.17)$ \\
\hline $\mathrm{VAR}_{W D}$ & $-0.16(0.21)$ & $0.04(0.21)$ & $-0.19(0.18)$ & $-0.14(0.23)$ & $0.10(0.19)$ & $-0.33(0.21)$ & $-0.17(0.16)$ \\
\hline VARWIDUR & $-0.22(0.22)$ & $0.28(0.20)$ & $-0.23(0.17)$ & $-0.43(0.26)$ & $0.26(0.19)$ & $-0.16(0.25)$ & $0.01(0.15)$ \\
\hline VAR WInVisits & $-0.16(0.21)$ & $-0.14(0.21)$ & $-0.15(0.18)$ & $-0.28(0.24)$ & $0.17(0.18)$ & $0.18(0.22)$ & $0.13(0.15)$ \\
\hline VAR $_{\text {WIRT }}$ & $0.21(0.23)$ & $0.27(0.23)$ & $-0.11(0.21)$ & $-0.05(0.30)$ & $-0.01(0.23)$ & $0.09(0.24)$ & $0.10(0.20)$ \\
\hline
\end{tabular}




\begin{tabular}{|c|c|c|c|c|c|c|c|}
\hline VAR $_{\text {WDRT }}$ & $0.12(0.23)$ & $0.07(0.24)$ & $0.00(0.21)$ & $-0.01(0.27)$ & $0.21(0.23)$ & $-0.02(0.24)$ & $0.14(0.20)$ \\
\hline \multicolumn{8}{|l|}{ Feed intake } \\
\hline $\mathrm{VAR}_{\mathrm{FI}}$ & $0.22(0.29)$ & $-0.23(0.31)$ & $0.14(0.25)$ & $-0.30(0.38)$ & $0.71(0.29)$ & $0.45(0.36)$ & $0.56(0.35)$ \\
\hline VAR $_{\text {FIDUR }}$ & $-0.21(0.20)$ & $-0.32(0.19)$ & $0.11(0.17)$ & $0.03(0.25)$ & $0.65(0.19)$ & $-0.17(0.20)$ & $-0.24(0.23)$ \\
\hline VAR $_{\text {FInVisits }}$ & $-0.19(0.12)$ & $-0.30(0.15)$ & $0.07(0.12)$ & $0.09(0.19)$ & $-0.01(0.15)$ & $0.04(0.17)$ & $0.05(0.11)$ \\
\hline VAR $_{\text {FIRT }}$ & $-0.73(1.74)$ & $-0.20(0.87)$ & $0.35(1.15)$ & $0.85(2.85)$ & $-0.82(1.48)$ & NA & NA \\
\hline \multicolumn{8}{|l|}{ Water intake } \\
\hline $\mathrm{OFF}_{\mathrm{WI}}$ & $-0.11(0.22)$ & $0.20(0.22)$ & $-0.04(0.20)$ & $-0.18(0.25)$ & $-0.29(0.21)$ & $-0.04(0.24)$ & $-0.16(0.18)$ \\
\hline $\mathrm{OFF}_{\mathrm{WD}}$ & $-0.34(0.23)$ & $-0.14(0.24)$ & $-0.25(0.20)$ & $-0.10(0.29)$ & $-0.21(0.23)$ & $-0.46(0.26)$ & $-0.37(0.18)$ \\
\hline OFF WIDUR $_{\text {W }}$ & $-0.18(0.19)$ & $0.06(0.23)$ & $-0.19(0.20)$ & $-0.46(0.32)$ & $0.27(0.22)$ & $-0.18(0.27)$ & $0.04(0.18)$ \\
\hline $\mathrm{OFF}_{\mathrm{WInVisits}}$ & $-0.07(0.17)$ & $-0.27(0.19)$ & $-0.02(0.18)$ & $-0.12(0.23)$ & $0.31(0.19)$ & $0.10(0.20)$ & $0.22(0.16)$ \\
\hline OFF $_{\text {WIRT }}$ & $0.38(0.27)$ & $0.23(0.26)$ & $-0.16(0.24)$ & $-0.08(0.34)$ & $-0.08(0.27)$ & $0.18(0.28)$ & $0.10(0.23)$ \\
\hline OFF $_{\text {WDRT }}$ & $0.31(0.28)$ & $0.18(0.29)$ & $-0.24(0.25)$ & $-0.30(0.30)$ & $-0.10(0.29)$ & $-0.13(0.30)$ & $-0.12(0.24)$ \\
\hline \multicolumn{8}{|l|}{ Feed intake } \\
\hline $\mathrm{OFF}_{\mathrm{FI}}$ & $-0.06(0.24)$ & $-0.45(0.21)$ & $0.38(0.19)$ & $0.51(0.23)$ & $0.27(0.24)$ & $0.28(0.30)$ & $0.48(0.25)$ \\
\hline OFF FIDUR $_{\text {Fid }}$ & $0.18(0.19)$ & $-0.16(0.19)$ & $0.26(0.17)$ & $0.08(0.21)$ & $-0.15(0.19)$ & $0.26(0.20)$ & $0.24(0.20)$ \\
\hline OFF FInVisits $_{\text {f }}$ & $-0.02(0.18)$ & $-0.03(0.22)$ & $0.20(0.18)$ & $0.14(0.25)$ & $0.23(0.24)$ & $0.32(0.23)$ & $0.34(0.17)$ \\
\hline $\mathrm{OFF}_{\mathrm{FIRT}}$ & $0.01(0.20)$ & $-0.36(0.23)$ & $0.28(0.19)$ & $0.48(0.24)$ & $0.60(0.27)$ & $-0.09(0.27)$ & NA \\
\hline
\end{tabular}

cNursery: challenge nursery; Combined: challenge nursery and finisher combined; ADWI: average daily water disappearance; ADWD:

average daily water dispensed; WIDUR: average daily water intake duration; WInVisits: average number of daily water intake visits;

WIRT: water disappearance rate; WDRT: water dispensed rate; ADFI: average daily feed intake; FIDUR: average daily feed intake duration; FInVisits: average number of daily feed intake visits; FIRT: feed intake rate; ADWI|ADFI, ADWD|ADFI, WIDUR|FIDUR,

WInVisits|FInVisits, ADFI|ADWI, ADFI|ADWD, FIDUR/WIDUR, FInVisits|WInVisits are the fitting the second trait as covariate for

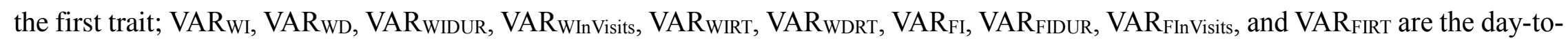




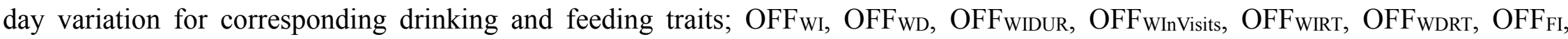
$\mathrm{OFF}_{\mathrm{FIDUR}}, \mathrm{OFF}_{\mathrm{FInVisits}}$, and $\mathrm{OFF}_{\mathrm{FIRT}}$ are the proportion of off days for corresponding drinking and feeding traits. 


\section{Supplementary figures:}

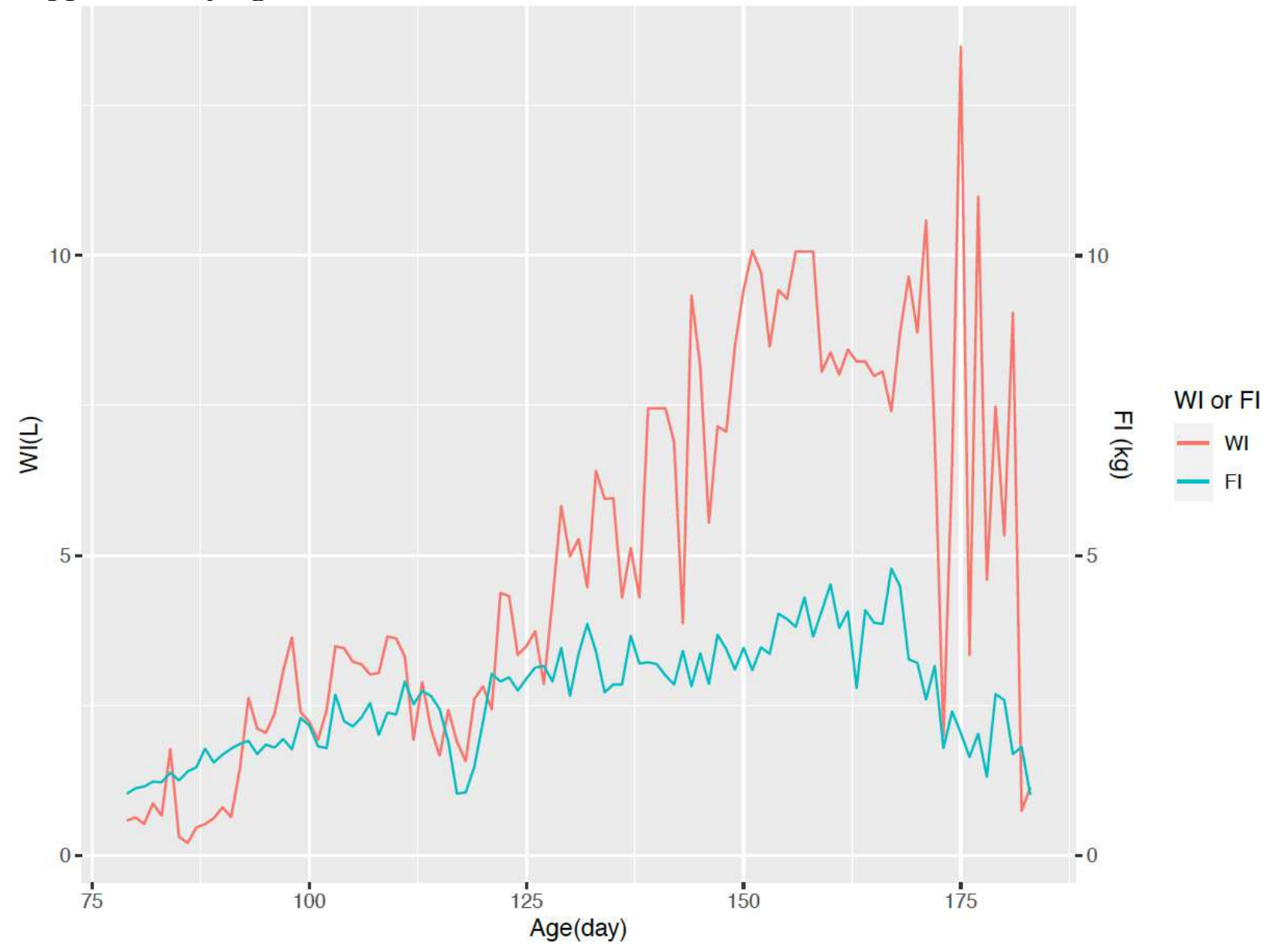

Figure S1. Raw daily feed intake (FI) and water disappearance (WI) for a randomly selected animal (0132). Both WI and FI had large day-to-day variation and had concurrent drops at around 120 and 160 days. 


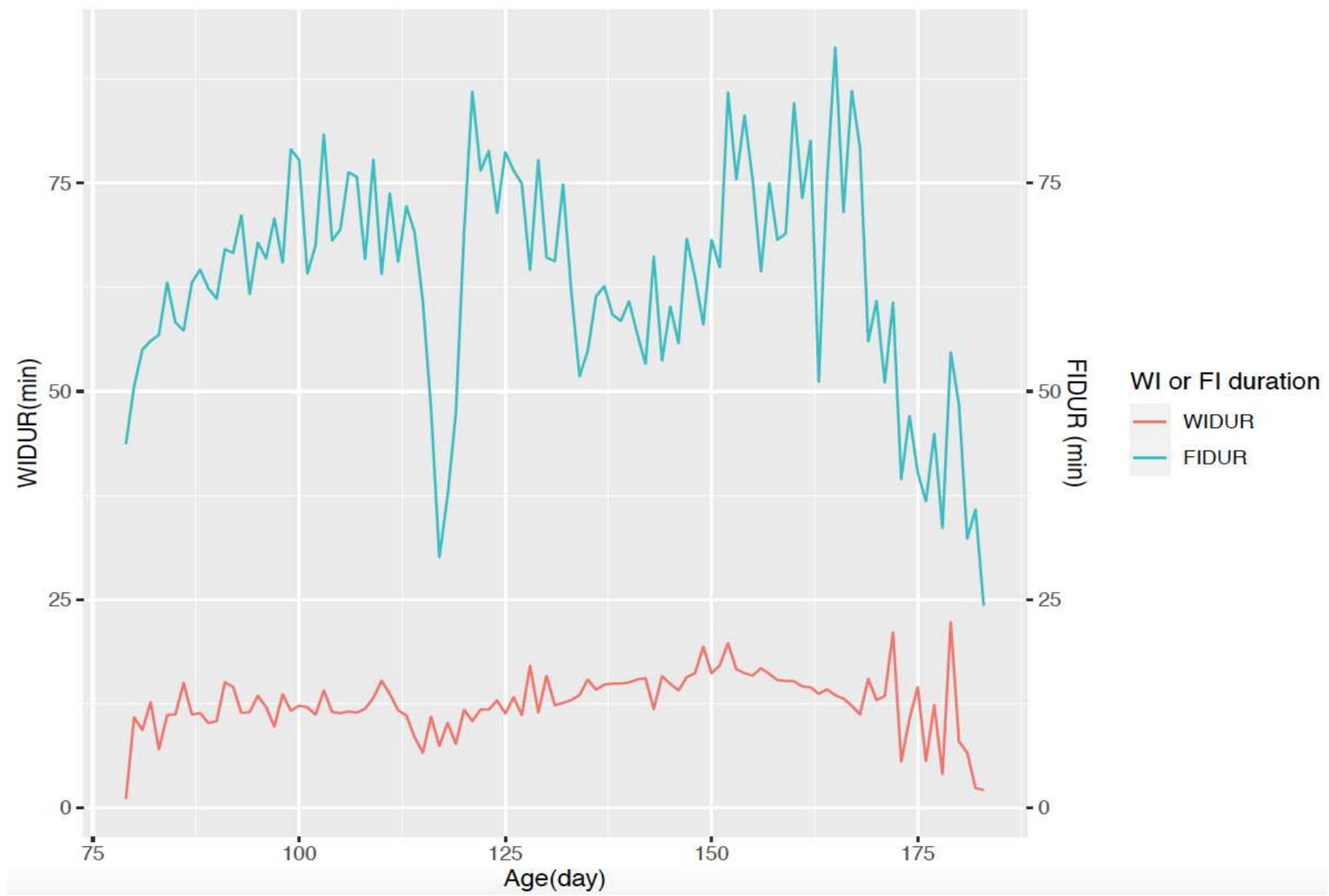

Figure S2. Raw daily feed intake duration (FIDUR) and water intake duration (WIDUR) for a randomly selected animal (0132). 


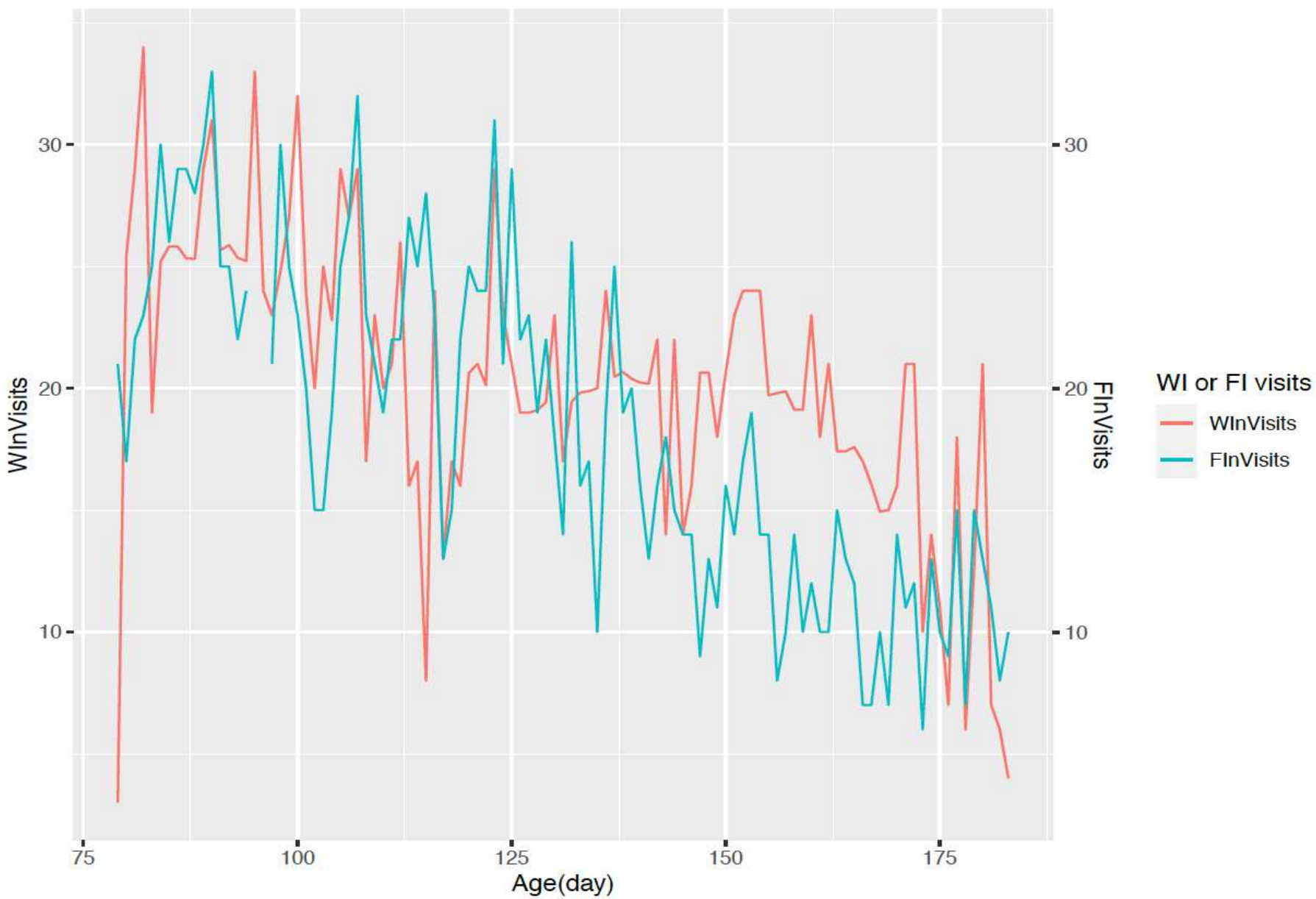

Figure S3. Raw daily feed intake visits (FInVisits) and water intake visits (WInVisits) for a randomly selected animal (0132). 


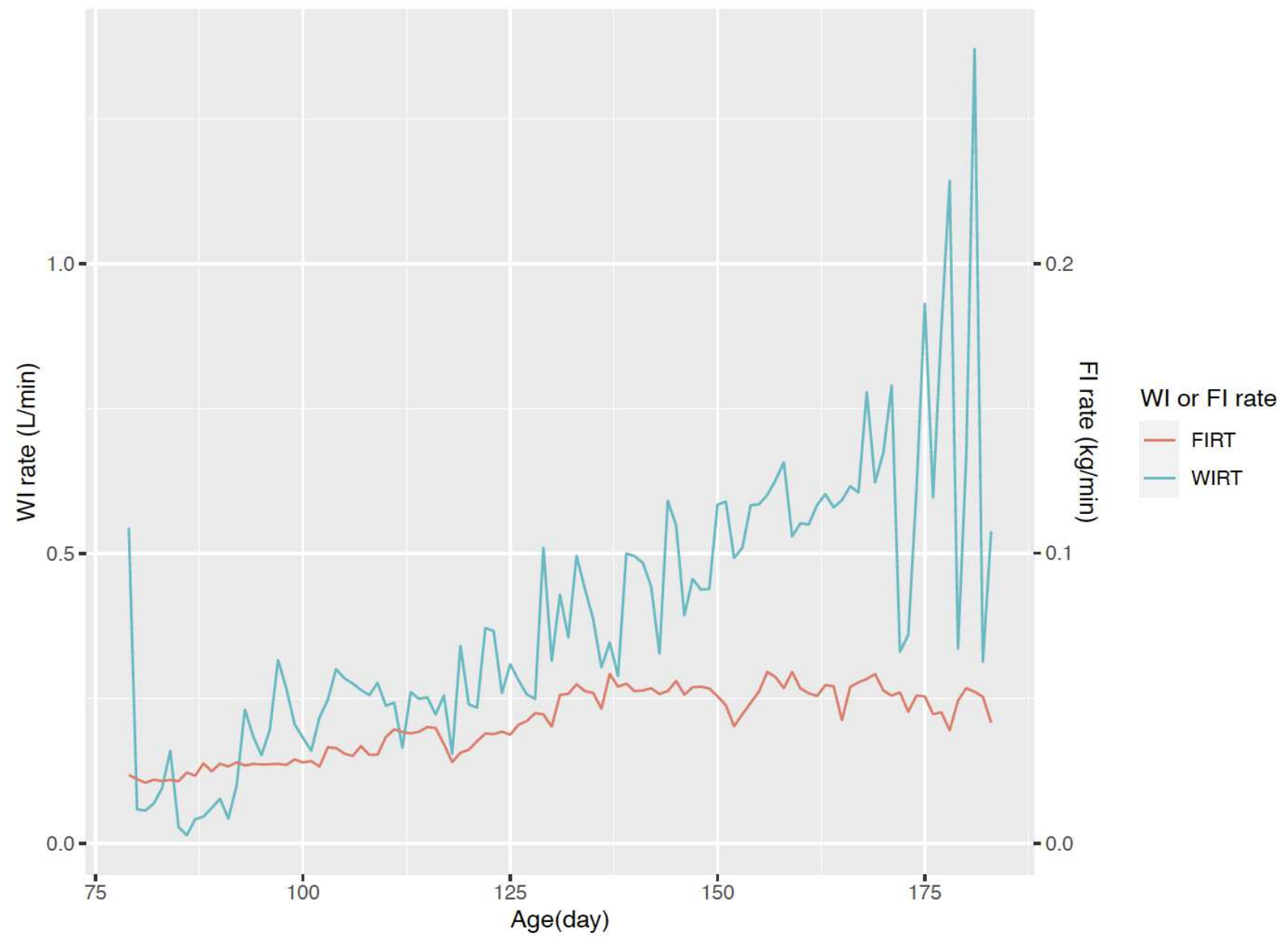

Figure S4. Raw daily feed intake rate (FIRT) and water disappearance rate (WIRT) for a randomly selected animal (0132). 


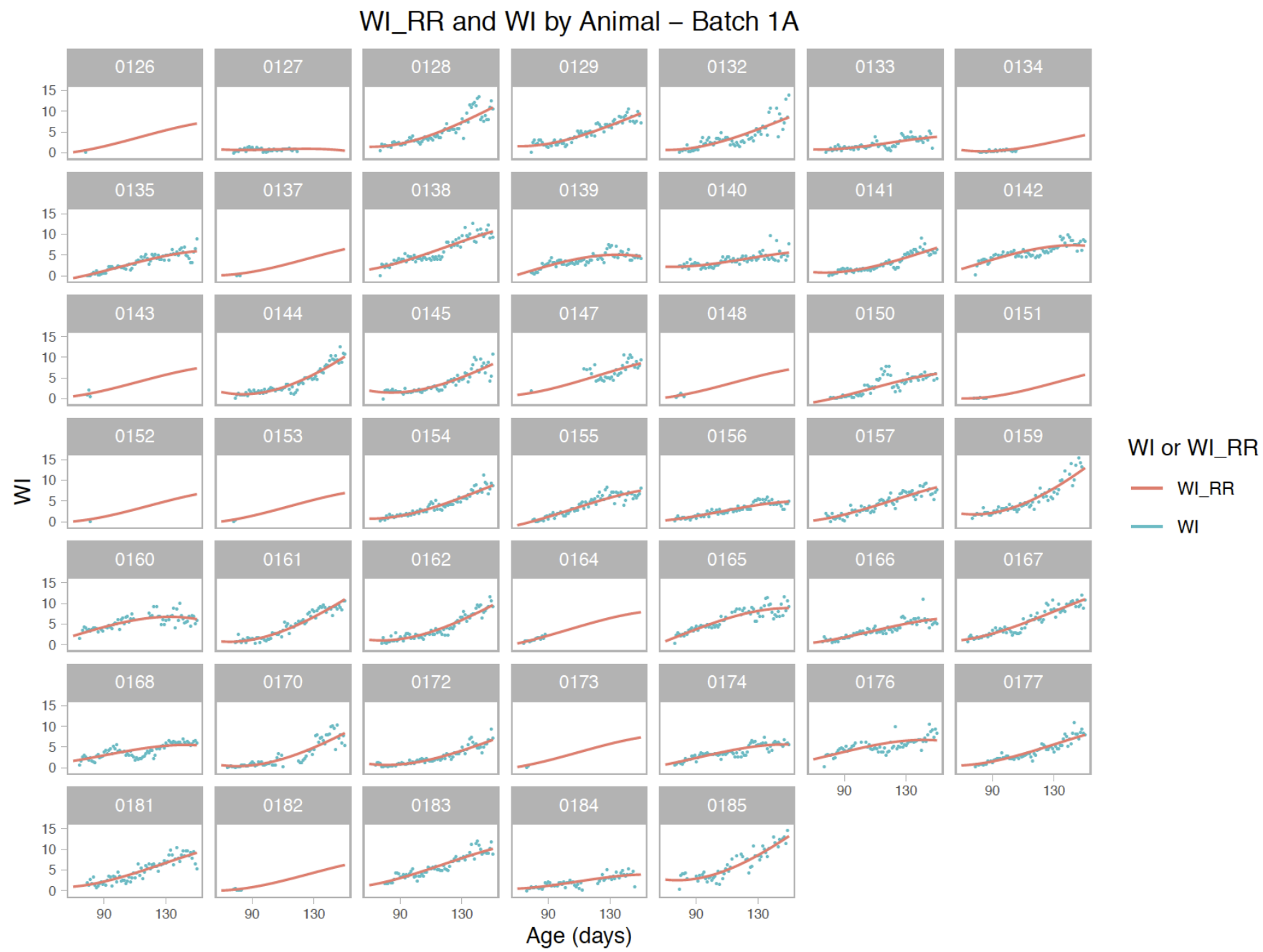

Figure S5. Raw water disappearance (WI) and predicted water disappearance (WI_RR) patterns defined using quadratic random regression model for for individual pigs in batch 1A. 


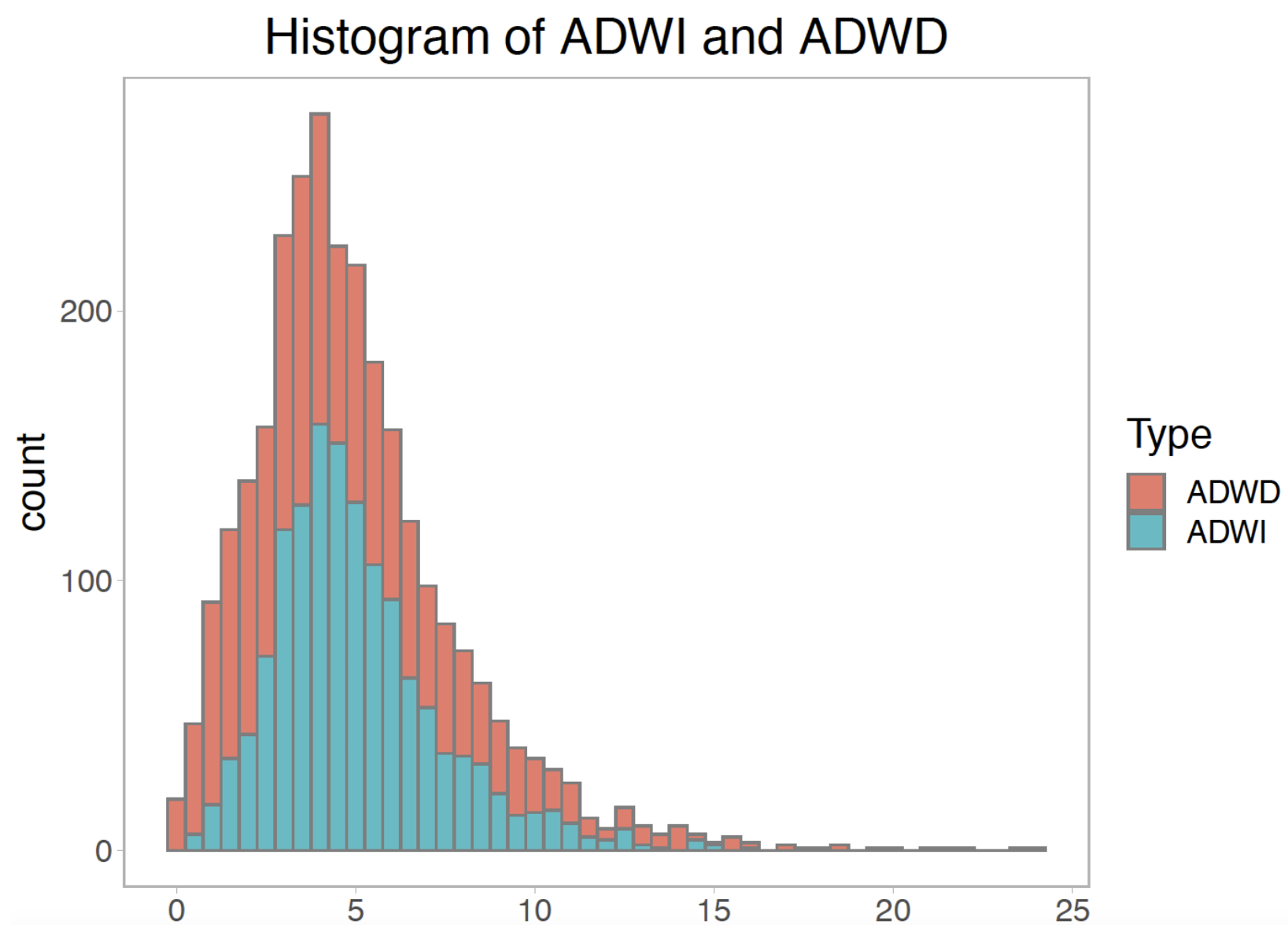

Figure S6. Histogram for average daily water disappearance (ADWI) and water dispensed (ADWD). 


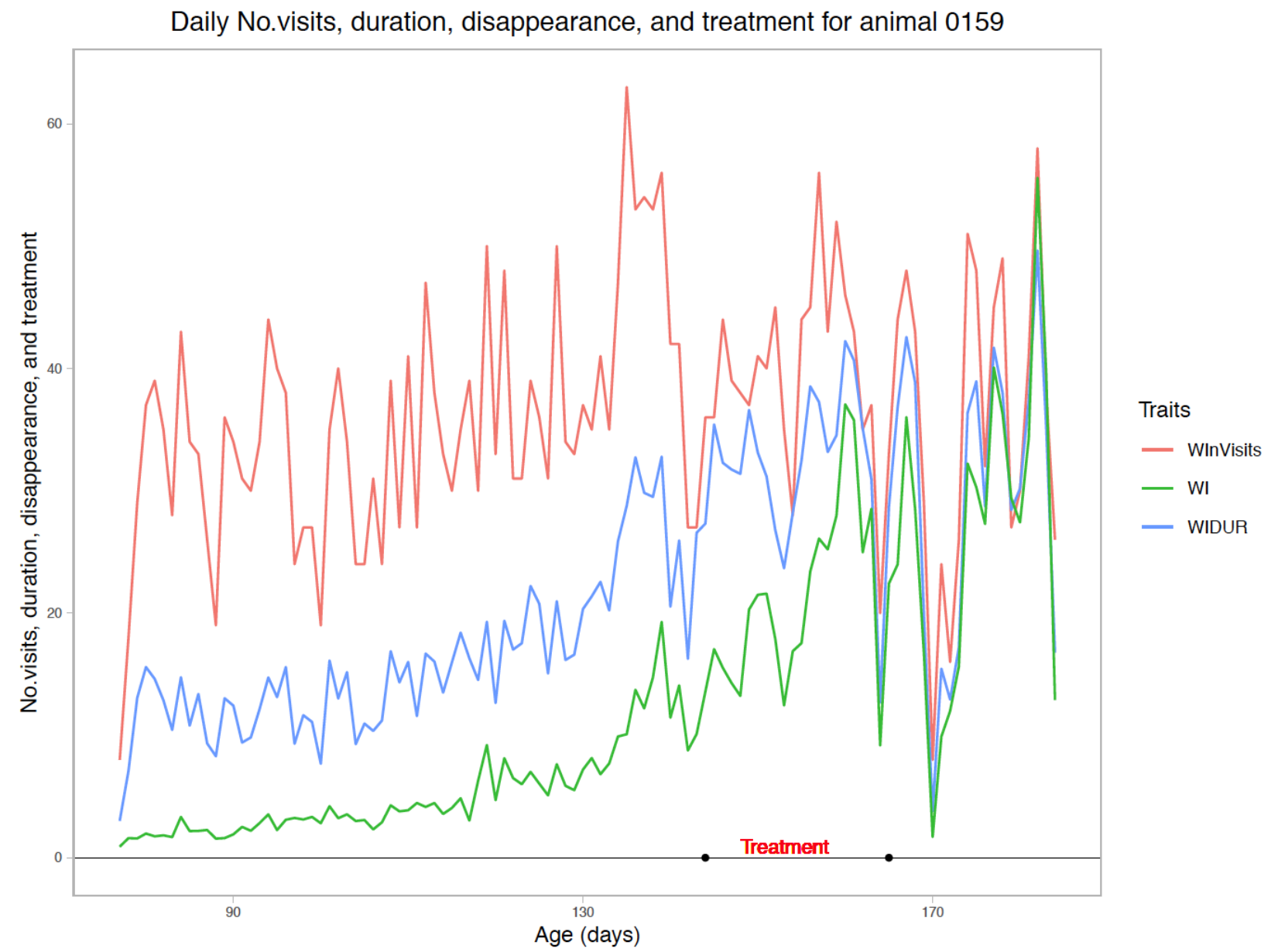

Figure S7. Raw daily water disappearance (WI), number of visits (WInVisits), and duration (WIDUR) for a randomly selected animal (0159). 
Figures

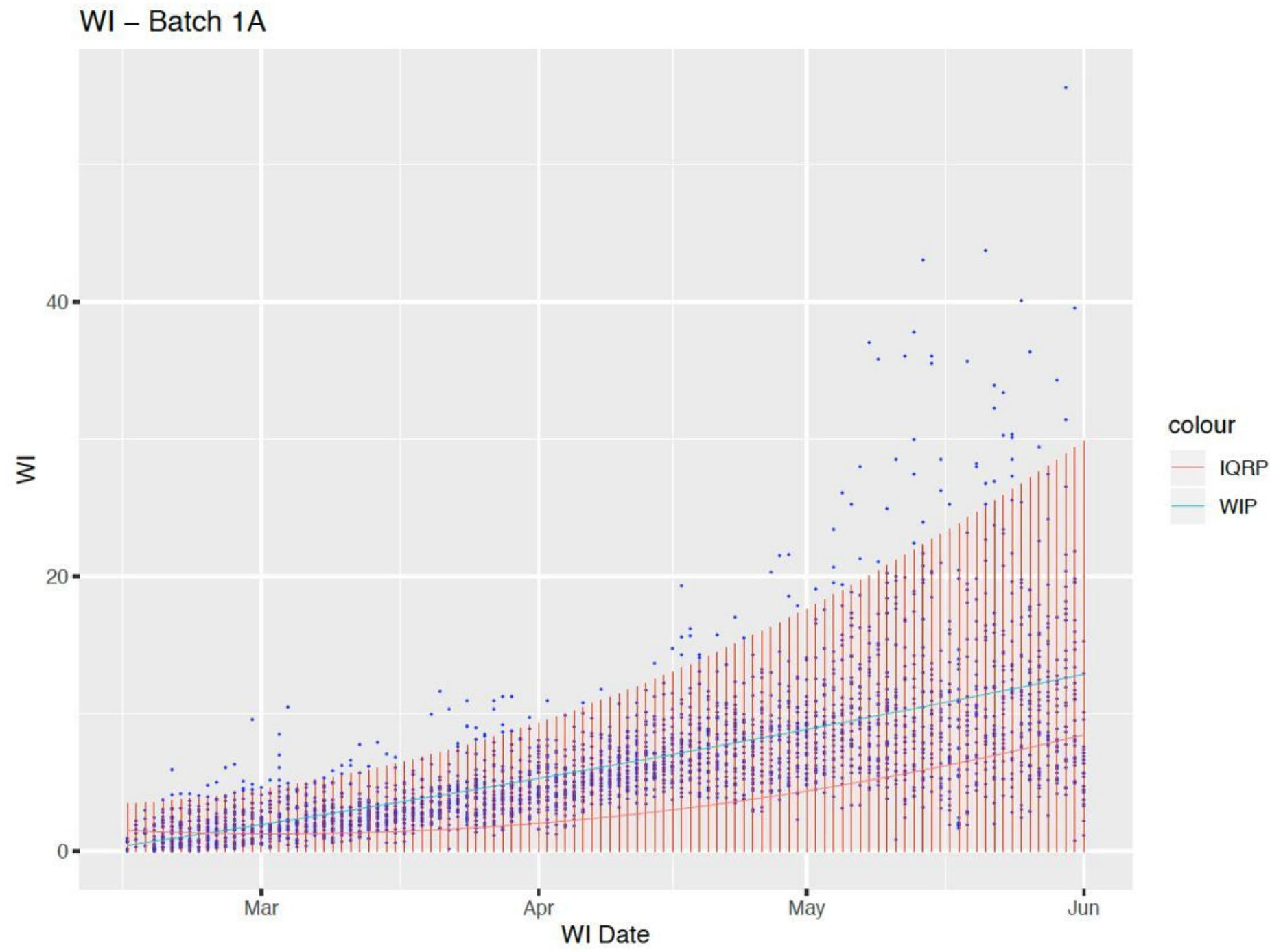

Figure 1

Outlier detection for water disappearance (WI) for a random batch of pigs based on predicted mean (WIP) +2 times the predicted interquartile range (IQRP). 


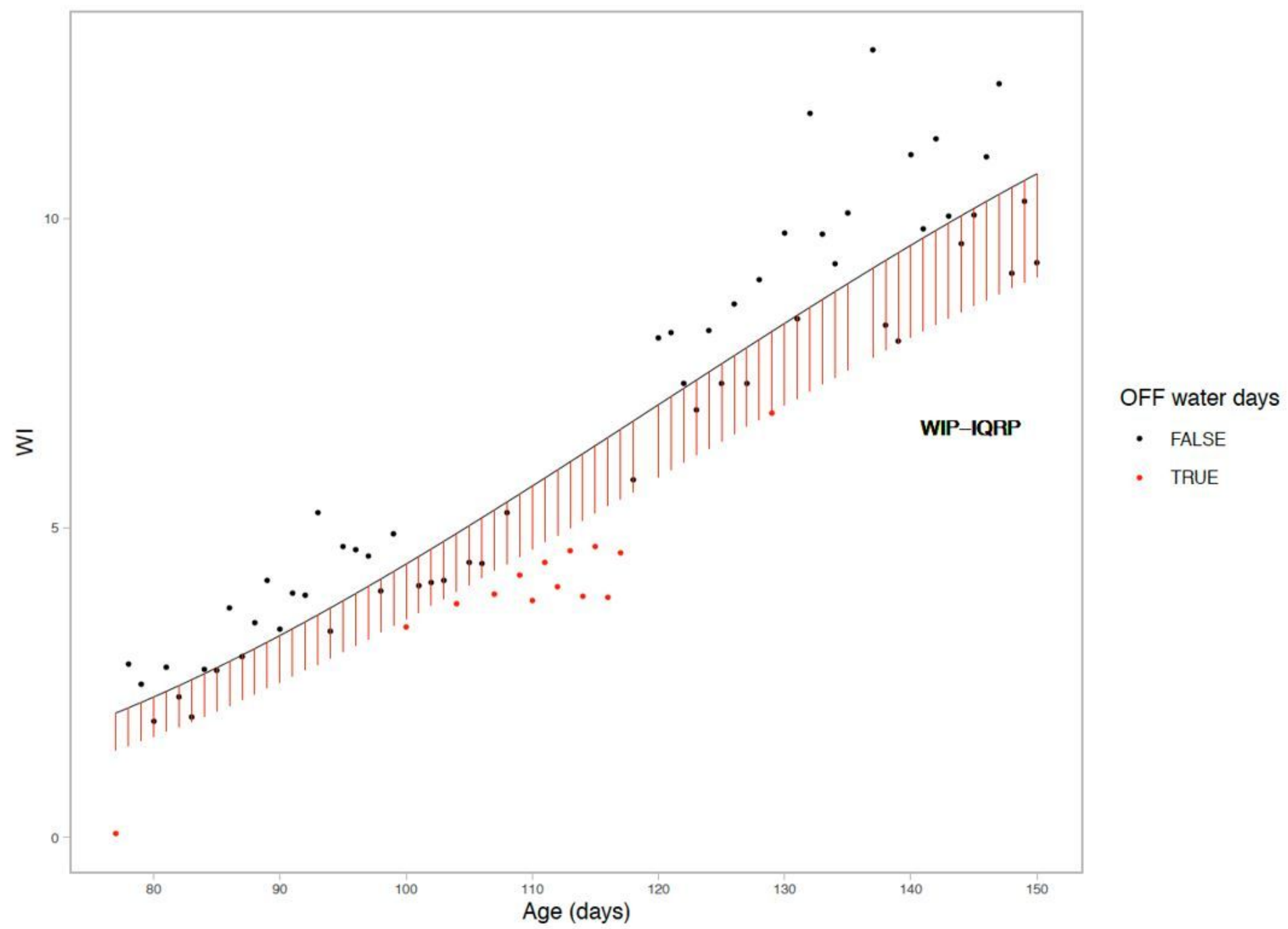

\section{Figure 2}

Derivation of day-to-day variation and proportion of off-water days for the water disappearance (WI) data of an randomly selected pig. TRUE: off-water days; FALSE: non off-water days. Solid line represents the predicted WI based on a quadratic random regression model; day-to-day variation in WI was computed as the square root of the sum of squared residuals. Red lines represent the predicted interquartile range of random regression residuals within the batch based on a quadratic regression model; daily water intake data that had residuals less than the predicted interquartile range were considered off-water days.

\section{Supplementary Files}

This is a list of supplementary files associated with this preprint. Click to download.

- sfig1.jpg

- sfig2.jpg 
- sfig3.jpg

- sfig4.jpg

- sfig5.jpg

- sfig6.jpg

- sfig7.jpg 